\title{
Structural failure during extrusion-based 3D printing processes
}

\author{
R. J. M. Wolfs ${ }^{1}$ - A. S. J. Suiker ${ }^{1}$ \\ Received: 18 February 2019 / Accepted: 1 May 2019 / Published online: 6 June 2019 \\ (C) The Author(s) 2019
}

\begin{abstract}
This contribution studies failure by elastic buckling and plastic collapse of wall structures during extrusion-based 3D printing processes. Results obtained from the parametric 3D printing model recently developed by Suiker (Int J Mech Sci, 137: 145-170, 2018), among which closed-form expressions useful for engineering practice, are validated against results of dedicated FEM simulations and 3D concrete printing experiments. In the comparison with the FEM simulations, various types of wall structures are considered, which are subjected to linear and exponentially decaying curing processes at different curing rates. For almost all cases considered, the critical wall buckling length computed by the parametric model turns out to be in excellent agreement with the result from the FEM simulations. Some differences may occur for the particular case of a straight wall clamped along its vertical edges and subjected to a relatively high curing rate, which can be ascribed to the approximate form of the horizontal buckling shape used in the parametric model. The buckling responses computed by the two models for a wall structure with imperfections of different wavelengths under increasing deflection correctly approaches the corresponding bifurcation buckling length. Further, under a specific change of the material properties, the parametric model and the FEM model predict a similar transition in failure mechanism, from elastic buckling to plastic collapse. The experimental validation of the parametric model is directed towards walls manufactured by $3 \mathrm{D}$ concrete printing, whereby the effect of the material curing rate on the failure behaviour of the wall is explored by studying walls of various widths. At a relatively low curing rate, the experimental buckling load is well described when the parametric model uses a linear curing function. However, the experimental results suggest the extension of the linear curing function with a quadratic term if the curing process under a relatively long printing time is accelerated by thermal heating of the $3 \mathrm{D}$ printing facility. In conclusion, the present validation study confirms that the parametric model provides a useful research and design tool for the prediction of structural failure during extrusion-based 3D printing. The model can be applied to quickly and systematically explore the influence of the individual printing process parameters on the failure response of $3 \mathrm{D}$-printed walls, which can be translated to directives regarding the optimisation of material usage and printing time.
\end{abstract}

Keywords Collapse behaviour $\cdot$ Elastic buckling $\cdot$ Plasticity $\cdot$ FEM modelling $\cdot 3 \mathrm{D}$ printing experiments ·

Effect of printing velocity · Thermal heating

\section{Introduction}

Extrusion-based 3D printing (E3DP) is an additive manufacturing technique, whereby the material is pushed in a molten, slurry or paste form through a nozzle to build up an

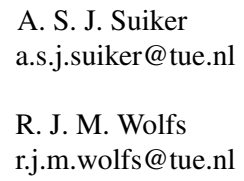

1 Department of the Built Environment, Eindhoven University of Technology, P.O. Box 513, NL-5600 MB, Eindhoven, The Netherlands object in a layerwise fashion along a calculated path. The specific layout of an individual layer is created by moving the nozzle in the horizontal plane at constant speed using robotic control. Once a layer is deposited, the extrusion nozzle, or the base on which the object is built, translates vertically across a distance equal to the layer height, after which the process is continued by placing a new layer on top of the previous layer, creating a so-called wall structure. Due to the curing of the layer, it binds to the layer beneath, such that the desired shape typically can be manufactured without the use of a support structure. Examples of E3DP are fused deposition modelling (also known as fused filament fabrication) [1-7], multiphase jet solidification [8], extrusion freeforming [9-11], contour crafting [12] and robocasting $[13,14]$, processes which mainly differ by 
the printing material applied, and by whether and how a heat source is used to bring the raw material to a printable state. In addition, E3DP bears similarities with the slip forming process applied in the building industry, where a concrete material is poured into a continuously moving form that permits the self-supported shaping of tall vertical structures (towers, bridges, buildings) and horizontal structures (roads) during the materials' initial curing phase $[15,16]$.

Over the past 20 years, E3DP has grown into the world's most widely used additive manufacturing technology, as a result of the low costs involved, its ability to produce complex geometrical shapes without the use of conventional tooling, the simplicity in operation, its suitability for low-volume production, a high dimensional accuracy, the easy integration with computed-aided design (CAD) software and the elimination of product storage costs $[4,7$, 17]. E3DP can be applied for creating models and prototypes, and for production applications, with examples in the aerospace industry (landing gear enclosure, jigs and fixtures, check gauges, aircraft components with high resistance to heat, moisture and chemicals), automotive industry (customised vehicle exteriors and interiors, complex parts for the engine compartment, vehicle scale models), food industry (pasta, meat-based products, chocolate), consumer goods industry (sporting goods, toys), pharmacokinetics (drug delivery devices), architectural and civil engineering (structural elements, bridges, houses), biomedical engineering (tissue scaffolding, implants, prosthetics), arms industry (gun prototyping), custom art and design (sculptures, modern abstract pieces), casting and tooling applications (production of dies and moulds, preforms, trim and drill tools) and electronics (sensors, electronic components and housing), among others (see [1, 3-7, 18-20] and references therein). The range of materials used in various E3DP processes is broad and includes thermoplastic polymers, biomaterials, cementitious materials, biological pastes, fibre-reinforced composites, mixtures of metal powders and polymeric binders, food pastes, ceramic powders, clays, gypsum, etc. [3, 4, 6, 7, 9-11, 14, 19, 20].

Despite the great success of E3DP, little is known about the influence of the manufacturing parameters and conditions on the mechanical behaviour of the object during the printing process. This is largely due to the complexity and diversity of the process parameters, as a result of which failures typically appear unexpectedly through a lack of mechanical performance or dimensional inaccuracy. Consequently, adequate printing process parameters commonly are established by means of trial and error, whereby it remains unclear if the optimal parameter set eventually is found under the conditions and requirements prescribed. This makes product development by 3D printing more expensive and time-consuming than necessary, in particular when the printed object is relatively large, such as in civil engineering and architectural applications. These aspects can be improved through the development of accurate mechanistic models, which are able to predict the influence of individual process parameters on the failure behaviour of the object during printing, thereby illustrating how the printing process can be optimised in terms of use of printing material and manufacturing time. The initially low strength and stiffness properties of the uncured printing material may ensure that the objects' failure resistance during manufacturing is more critical than during operation; hence, minimising the amount of printing material needed to maintain the strength and stability of the object during the printing process may significantly reduce the production costs.

Recently, Suiker [21] developed a mechanistic parametric model that can be used for the prediction of structural failure of straight, free-standing walls generated during E3DP processes. The model focuses on the competition between elastic buckling and plastic collapse (see Fig. 1), which typically are identified as the two most relevant structural failure mechanisms during E3DP processes (see also [22-25]). The elastic buckling mechanism reflects failure caused by a loss of geometrical stability, while plastic collapse is characterised by the maximum stress (at the bottom of the wall) reaching the material yield strength. The parametric model in [21] reveals the sensitivity of these failure mechanisms to the actual printing process parameters, which are the curing properties of the printing material, the printing velocity, the geometrical features of the printed object, the heterogeneous strength and stiffness characteristics of the object, the non-uniform dead weight loading and the presence of imperfections. It is demonstrated that the relatively large number of parameters characterising the $3 \mathrm{D}$ printing process can be reduced to five unique, dimensionless (time and length scale) parameters, with three parameters defining elastic buckling and two parameters representing plastic collapse. In addition, closed-form expressions are presented for the critical buckling length

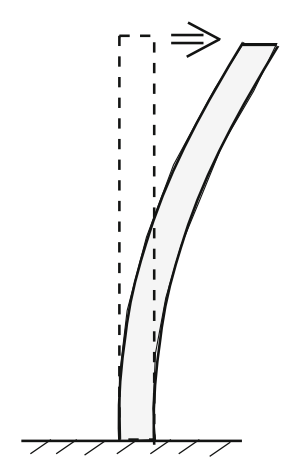

Elastic buckling

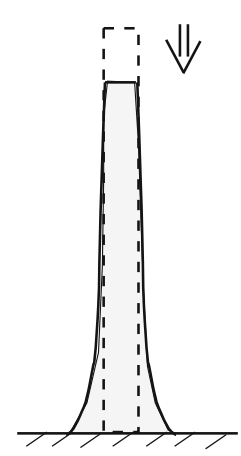

Plastic collapse
Fig. 1 Wall failure by elastic buckling (left) and plastic collapse (right) 
and plastic collapse length of a free wall (i.e., a wall without support along its vertical edges), and design graphs are provided for the critical failure lengths of simply supported and fully clamped walls. It is further demonstrated how the parametric model can be applied for determining the failure behaviour of more complicated wall geometries, such as a rectangular wall-layout. Finally, the buckling behaviours calculated for the free wall and the rectangular wall layout are compared to the experimental results of 3Dprinted concrete wall structures, which turn out to be in good agreement.

In order to validate the accuracy of the parametric 3D printing model and support the implementation of the design formulas and graphs presented in [21] in engineering practice, it is useful to compare results of this model to those obtained by dedicated finite element method (FEM) analyses and supplementary $3 \mathrm{D}$ printing experiments. A design parameter that deserves special attention in this comparison study is the material curing rate, or, analogously, the printing velocity, since its optimisation straightforwardly leads to a reduction in printing time, and thus to a decrease of the manufacturing costs. Accordingly, in the present communication for the various straight wall structures mentioned above, the failure response computed by the parametric $3 \mathrm{D}$ printing model is validated against results obtained by dedicated FEM analyses and 3D printing experiments, both carried out at various curing rates. The finite element method serves as an excellent tool for the accurate modelling of 3D printing processes of structures of arbitrary geometry, as recently demonstrated in [24] for 3D-printed cylindrical walls collapsing by non-linear buckling. The experimental validation is directed towards free walls manufactured by 3D concrete printing, whereby the effect of the material curing rate on the failure behaviour of the wall is explored by considering walls of various widths. The validation study includes the linear and exponentially decaying, time-dependent curing processes analysed in [21], and suggests the application of a quadratic curing function if the curing process under a relatively long printing time is accelerated by thermal heating of the 3D printing facility.

The manuscript is organised as follows. In Section 2, the main equations and characteristics of the mechanistic parametric model presented in [21] are outlined, thereby distinguishing between failure due to elastic buckling and plastic collapse. Section 3 starts by comparing the elastic buckling response computed by the parametric model for the free wall, simply supported wall and fully clamped wall to the results calculated by FEM modelling, assuming a linear curing process and considering three different curing rates (or printing velocities). Subsequently, for the free wall configuration, the buckling response under an exponentially decaying curing process is analysed, followed by a study on the influence of imperfections. The comparison study is continued with analyses of the elastic buckling response of a rectangular wall layout, and the transition from elastic buckling to plastic collapse under a specific change of the material parameters. In Section 4, the results of the parametric model are validated against experimental results obtained by the $3 \mathrm{D}$ printing of concrete wall structures. The influence of the printing velocity on the buckling behaviour of the free wall is analysed by choosing three different wall widths. In addition, the effect by thermal heating of the 3D printing facility on the buckling response is highlighted. Section 5 presents the main conclusions of the validation study.

\section{Review of parametric 3D printing model}

The mechanical stresses during the 3D printing of a vertical, free-standing wall structure monotonically increase as a result of a growing dead weight loading. At some critical moment, this may lead to wall failure by either elastic buckling (a stability mechanism) or plastic collapse (a strength mechanism) (see Fig. 1). These two basic failure mechanisms form the basis of the parametric 3D printing model recently developed by Suiker [21], of which the main characteristics and equations are outlined in this section.

Consider the three basic configurations of a printed wall of length $l$, width $b$ and thickness $h$ illustrated in Fig. 2, which have been taken from [21]. These configurations differ by the boundary conditions in the (horizontal) $y$ direction, and accordingly are designated as (i) a free wall, (ii) a simply supported wall and (iii) a fully clamped wall. The walls are printed by adding material in a layer-wise fashion, whereby it is assumed that during the printing of an individual layer the strength and stiffness properties in that layer do not significantly alter. In other words, the characteristic time defining the curing process of the printing material is considered to be larger than the period needed for the printing of an individual layer. In various E3DP processes, this condition is of vital importance from a structural point of view, since it supports a good bonding between the actual layer and the layer beneath. Accordingly, the spatial variations in strength and stiffness as a result of the curing behaviour of the printing material may be assumed to occur solely along the direction of increasing wall length, i.e. the $x$-direction in Fig. 2.

\subsection{Elastic buckling}

In accordance with plate theory, for the description of elastic buckling the out-of-plane deflection $w=\hat{w}(x, y)$ of the wall structures illustrated in Fig. 2 may be subjected to a separation of variables:

$w=\hat{w}(x, y)=\hat{w}^{c}(x) \hat{f}(y)$, 
Free wall

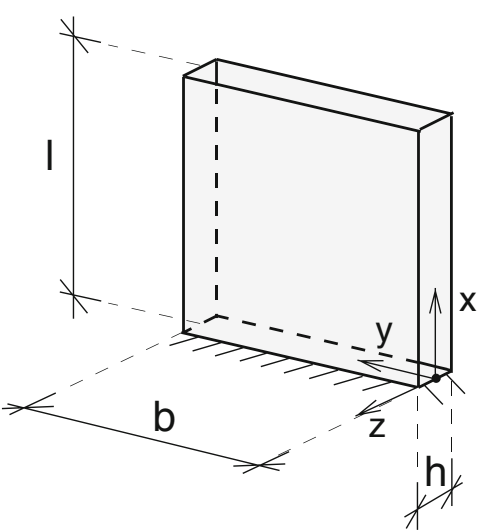

Simply-supported wall

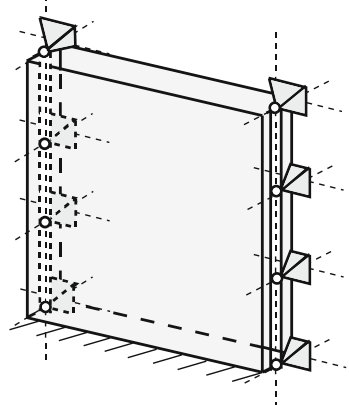

Fully-clamped wall

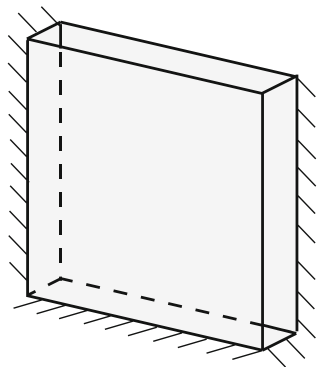

Fig. 2 Three basic wall configurations: a free wall (left), a simply supported wall (middle) and a fully clamped wall (right). The figure has been reprinted from [21]

where $\hat{w}^{c}(x)$ is the out-of-plane displacement along the (vertical) $x$-direction of the wall, evaluated at the symmetry line $y=b / 2$, and $\hat{f}(y)$ designates the normalised out-ofplane displacement along the (horizontal) $y$-direction. For the three wall types sketched in Fig. 2, the fundamental horizontal buckling shapes satisfying the corresponding horizontal boundary conditions are assumed as:

Free wall:

$$
\begin{aligned}
& \hat{f}(y)=1, \\
& \hat{f}(y)=\sin (\pi y / b), \\
& \hat{f}(y)=(1-\cos (2 \pi y / b)) / 2 .
\end{aligned}
$$

As demonstrated in [21], after combining Eq. 1 with the horizontal buckling shape in Eq. 2, the equilibrium equation and boundary conditions to be solved for wall buckling can be entirely formulated in terms of the unknown out-of-plane displacement along the $x$-direction, $w^{c}=\hat{w}^{c}(x)$.

The growth of a wall of length $l$ in $x$-direction is considered to be a continuous process that occurs at a constant wall growth velocity. The wall growth velocity $i$ with the superimposed dot indicating the time derivativecan be expressed in terms of the actual printing process parameters as:

$i=\frac{Q}{v_{n} h T_{l}}$,

where $Q$ represents the material volume discharged from the printing nozzle per unit time, $v_{n}$ is the horizontal velocity of the printing nozzle, $T_{l}$ is the period required for printing an individual material layer and $h$ is the thickness of the wall. Due to curing, the stiffness of the printing material evolves with time, which can be formally expressed as

$\hat{E}_{*}(t)=\hat{g}_{*}(t) E_{0}$, where $g_{*}=\hat{g}_{*}(t)$ is the characteristic curing function and $E_{0}$ is the initial stiffness of the printing material, corresponding to the moment the material is discharged from the printing nozzle. In [21], two basic types of curing functions are considered, namely a linear curing function $g_{*}=\hat{g}_{*}^{l}(t)$, whereby:

$\hat{g}_{*}^{l}(t)=1+\xi_{E}^{l} t$,

and an exponentially decaying curing function $g_{*}=\hat{g}_{*}^{e}(t)$, with:

$\hat{g}_{*}^{e}(t)=\gamma_{E}+\left(1-\gamma_{E}\right) \exp \left(-\xi_{E}^{e} t\right) \quad$ where $\quad \gamma_{E}=\frac{E_{\infty}}{E_{0}}$.

Here, $\xi_{E}^{l}$ and $\xi_{E}^{e}$ are the curing rates for the elastic modulus (with dimension of time ${ }^{-1}$ ) in the linear and exponential evolutions, respectively. Further, $\gamma_{E}$ is the ratio between the final stiffness $E_{\infty}$, obtained when $t \rightarrow \infty$, and the initial stiffness $E_{0}$ in the exponential curing process. The characteristics of the linear and exponentially decaying curing functions are illustrated in Fig. 3.

The exponential form is representative of a strongly accelerating curing process that quickly leads to a plateau value for the stiffness (and/or the strength), as realised under the application of an external stimulus, e.g. UV light or heat $[26,27]$, or through the addition of a chemical agent [28].

The specific time evolution of the macroscopic material properties is related to microstructural characteristics of the printing material, including the morphology, domain size and size homogeneity [29]. It should be mentioned that these aspects in general may be influenced by the process conditions applied during extrusion-based 3D printing. For example, for polypropylene and polycarbonate composites, the deposition orientation and deposition speed applied during the printing process may have a strong effect on the morphological structure of the printing material, and thus on its mechanical properties, the level of material anisotropy 


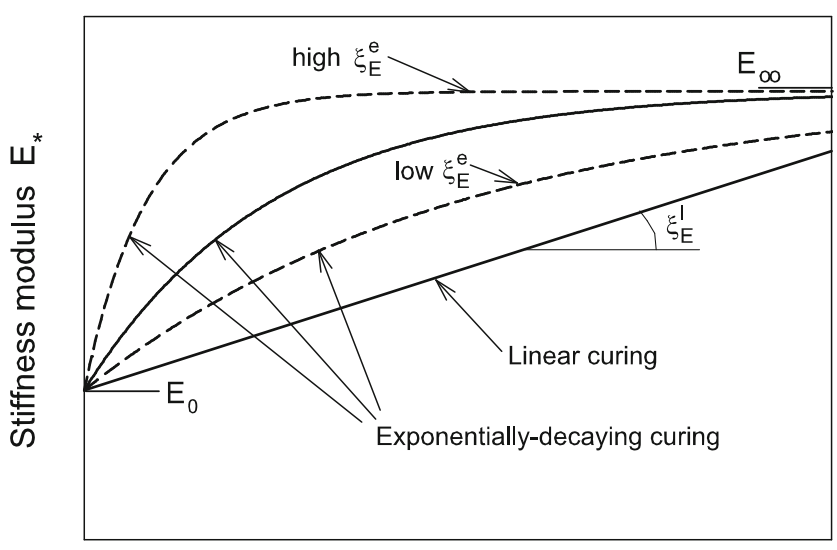

0

\section{Curing time $t$}

Fig. 3 Characteristics of the linear and exponentially decaying curing functions for the stiffness modulus $E_{*}$ (solid lines), in accordance with Eqs. 4, 5 and 6. The dashed lines designate exponentially decaying curing functions characterised by relatively high and low curing rates $\xi_{E}^{e}$. The figure has been reprinted from [21]

and the surface roughness of the printed object [29, 30]. Additional morphological changes in a thermoplastic polymer printing material occur when melting the feedstock and pushing it through the printing nozzle, which are caused by the vaporisation of adsorbed water and the breakage of polymer chains under the application of relatively high temperatures [31]. When applying Eq. 4 for calibrating the time-dependent stiffness properties from experiments, the above aspects should be carefully considered and, when relevant, be taken into account. For the specific case of $3 \mathrm{D}$ concrete printing, however, the significance of these aspects is relatively low, which allows calibrating Eq. 4 from material tests that are independent of the printing process conditions mentioned above (see also Section 4.1). In case the concrete printing material is reinforced by fibres, the microstructural stiffening effect by the fibres should be accounted for in the calibration of Eq. 4, although the influence may be negligible for low fibre volume percentages [32].

As shown in [21], for the incorporation of time-related process parameters in the parametric model, i.e. the material curing rate and the printing velocity, it is convenient to develop the model equations in an Eulerian coordinate system that is attached to the printing nozzle. In accordance with Fig. 4, the Eulerian coordinate $X$ is related to the Lagrangian coordinate $x$ connected to the bottom of the printed wall as:

$X=\hat{X}(x, t)=x-l=x-i t$,

with $t$ denoting time. The Eulerian coordinate $X$ can be expressed into a dimensionless form $\bar{X}$ (with the

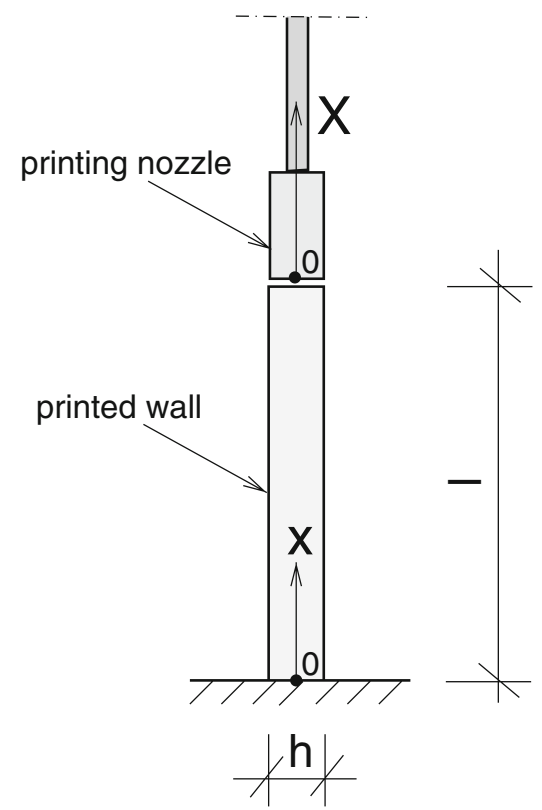

Fig. 4 A Lagrangian coordinate system $x$ with its origin connected to the bottom of the printed wall, and an Eulerian coordinate system $X$ with its origin connected to the end of the printing nozzle. The figure has been reprinted from [21]

superimposed bar emphasising that the parameter is dimensionless) as follows:

$\bar{X}=\hat{\bar{X}}(x, t)=\frac{\xi_{E}}{i} X$,

with $\xi_{E}$ representing the curing rate of the elastic stiffness $E_{*}$ of the printing material. Similar to Eq. 4, the evolution of the elastic stiffness during curing may be formally expressed as a function of the Eulerian coordinate $\bar{X}$ as:

$\hat{E}_{*}(\bar{X})=\hat{\bar{g}}_{*}(\bar{X}) E_{0}$,

in which $\bar{g}_{*}=\hat{\bar{g}}_{*}(\bar{X})$ thus reflects the specific curing function adopted. Via the coordinate transformation given by Eqs. 7 and 8, the linear curing function, Eq. 5, in terms of $\bar{X}$ becomes:

$\hat{\bar{g}}_{*}^{l}(\bar{X})=1-\bar{X} \quad$ with $\quad \bar{X}=\frac{\xi_{E}^{l} X}{i}$,

and the exponentially decaying curing function, Eq. 6, turns into:

$$
\begin{aligned}
\hat{\bar{g}}_{*}^{e}(\bar{X})= & \gamma_{E}+\left(1-\gamma_{E}\right) \exp (\bar{X}) \\
& \text { with } \quad \bar{X}=\frac{\xi_{E}^{e} X}{i} \quad \text { and } \quad \gamma_{E}=\frac{E_{\infty}}{E_{0}} .
\end{aligned}
$$

As a next step, the out-of-plane displacement is henceforth expressed in a dimensionless form, $\bar{w}^{c}=w^{c} / h$, with $h$ the wall thickness. In accordance with the coordinate transformation given by Eqs. 7 and 8, $\bar{w}^{c}$ can be expressed 
as a function of the dimensionless Eulerian coordinate $\bar{X}$, and may be subsequently decomposed as:

$\hat{\bar{w}}^{c}(\bar{X})=\hat{\bar{w}}^{c, 0}(\bar{X})+\hat{\bar{w}}^{c, F}(\bar{X})$,

in which the displacement function $\bar{w}^{c, 0}$ characterises possible geometrical imperfections generated during printing of the wall, and $\bar{w}^{c, F}$ is the deflection under the applied loading $F$ (i.e. the dead weight loading of the wall). The equilibrium condition for elastic buckling can be expressed in terms of these two displacement components via the following non-homogeneous fourth-order differential equation [21]:

$$
\begin{aligned}
& \left(\bar{g}_{*} \bar{w}_{, \bar{X} \bar{X}}^{c, F}\right)_{, \bar{X} \bar{X}}-\left(\bar{k}_{1} \bar{w}_{, \bar{X}}^{c, F}\right)_{, \bar{X}}+\bar{k}_{2} \bar{w}^{c, F} \\
& =\left(\bar{k}_{1}^{r} \bar{w}_{, \bar{X}}^{c, 0}\right)_{, \bar{X}}-\bar{k}_{2}^{r} \bar{w}^{c, 0} .
\end{aligned}
$$

Under vanishing imperfections the right-hand side of the above differential equation becomes 0 ; this homogeneous differential equation can be used for computing the critical bifurcation buckling length of the wall. The curing function $\bar{g}_{*}=\hat{\bar{g}}_{*}(\bar{X})$ is given by Eq. 10 for linear curing and by Eq. 11 for exponentially decaying curing, while the other functions read ${ }^{1}$

$$
\begin{aligned}
\bar{k}_{1} & =\hat{\bar{k}}_{1}(\bar{X})=\lambda \bar{X}+2 c_{y *}\left(\frac{n_{y *} \pi}{\epsilon}\right)^{2} \bar{g}_{*}, \\
\bar{k}_{2} & =\hat{\bar{k}}_{2}(\bar{X})=c_{y *}\left(\frac{n_{y *} \pi}{\epsilon}\right)^{4} \bar{g}_{*} \\
& +c_{y *}\left(\frac{n_{y *} \pi}{\epsilon}\right)^{2}\left(K_{y *} \lambda \bar{X}-v\left(\bar{g}_{*}\right)_{, \bar{X}}\right), \\
\bar{k}_{1}^{r} & =\hat{\bar{k}}_{1}^{r}(\bar{X})=\lambda \bar{X} \\
\bar{k}_{2}^{r} & =\hat{\bar{k}}_{2}^{r}(\bar{X})=c_{y *}\left(\frac{n_{y *} \pi}{\epsilon}\right)^{2} K_{y *} \lambda \bar{X},
\end{aligned}
$$

with the dimensionless parameters $\lambda$ and $\epsilon$ given by:

$\lambda=\frac{\rho g h}{D_{0}}\left(\frac{i}{\xi_{E}}\right)^{3}, \quad \epsilon=\frac{\xi_{E} b}{i}$.

The parameter $\lambda$ includes the dead weight loading characteristics via the density $\rho$, the gravitational acceleration $g$, and the initial wall bending stiffness,

$D_{0}=\frac{E_{0} h^{3}}{12\left(1-v^{2}\right)}$,

where $v$ is the Poisson ratio of the printing material (which is assumed to be constant during the curing process) and $h$ is the wall thickness. The function $K_{y *}=\hat{K}_{y *}(\bar{X})$ appearing in Eq. 15 represents the coefficient of lateral stress, which quantifies the horizontal stress generated in the wall by the vertical dead weight loading; this function

\footnotetext{
${ }^{1}$ In Eq. 15, the asterisk subindex is used to emphasise that the actual parameter is a function of the dimensionless Eulerian coordinate $\bar{X}$.
}

takes values between 0 (for a wall without in-plane support along the vertical boundaries) and $v$ (for a wall with inplane support along the vertical boundaries) [21]. Finally, the parameters $n_{y *}$ and $c_{y *}$ present in Eq. 15 respectively reflect the number of half-waves characterising the buckling shape in $y$-direction, and a constraint factor that depends on the type of boundary conditions applied in the $y$-direction. Specifically, for the three basic wall types depicted in Fig. 2, $n_{y *}$ and $c_{y *}$ have the following values [21]:

Free wall:

$$
\begin{aligned}
& n_{y *}=0, \quad c_{y *}=0, \\
& n_{y *}=1, \quad c_{y *}=1,
\end{aligned}
$$

$$
\text { Fully clamped wall: } \quad n_{y *}=2, \quad c_{y *}=0.5 \text {. }
$$

Note that for the simply supported wall, the rotation about the vertical axis at the boundaries in $y$-direction is fully free, whereas for the fully clamped wall it is completely constrained. When this rotation is partly constrained by a rotational stiffness that is non-uniform along the $\bar{X}$-direction of the wall boundaries, $\bar{k}_{r}=\hat{\bar{k}}_{r}(\bar{X})$, the parameters $n_{y *}$ and $c_{y *}$ fall within ranges bounded by the above-mentioned values for the simply supported and fully clamped walls, i.e. $1 \leq n_{y *} \leq 2$ and $1 \geq c_{y *} \geq 0.5$, and are formally expressed by the functions:

$$
\begin{aligned}
& n_{y *}=\hat{n}_{y *}(\bar{X})=\hat{n}_{y *}\left(\hat{\bar{k}}_{r}(\bar{X})\right), \\
& c_{y *}=\hat{c}_{y *}(\bar{X})=\hat{c}_{y *}\left(\hat{\bar{k}}_{r}(\bar{X})\right) .
\end{aligned}
$$

As demonstrated in [21], for structural layouts composed of multiple straight walls, the rotational stiffness $\bar{k}_{r}$ may effectively account for the constraining influence of a secondary, supporting wall on the primary wall that buckles. By deriving the function $\hat{\bar{k}}_{r}(\bar{X})$ for such a wall geometry, and inserting the result into the following closed-form expressions [21]:

$$
\begin{aligned}
n_{y *} & =\hat{n}_{y *}\left(\bar{k}_{r}\right)=1.984\left[1-\exp \left(-\left(0.360 \bar{k}_{r}+0.430\right)^{0.452}\right)\right], \\
c_{y *} & =\hat{c}_{y *}\left(\bar{k}_{r}\right)=0.5+0.309 \exp \left(-0.854 \bar{k}_{r}\right) \\
& +0.192 \exp \left(-0.183 \bar{k}_{r}\right),
\end{aligned}
$$

the functions, Eq. 18, become specified. In this way, it is possible to analyse the buckling behaviour of wall structures with more advanced geometries than those presented in Fig. 2 (see Section 3.4 for an example).

In addition to the buckling equation, Eq. 13, the boundary conditions in the $\bar{X}$-direction need to be formulated. In line with Eqs. 7 and 8, the locations of the Eulerian boundaries are $\bar{X}=-\kappa$ (bottom of the wall) and $\bar{X}=0$ (top of the wall), with

$\kappa=\frac{\xi_{E} l}{i}$. 
At the bottom of the wall, $\bar{X}=-\kappa$, the wall structure is fully clamped, in correspondence with the boundary conditions [21]:

$\bar{w}^{c}=0$,

$\bar{w}_{, \bar{X}}^{c}=0$,

while at the top of the wall, $\bar{X}=0$, the wall structure can displace and rotate freely, in accordance with [21]:

$$
\begin{aligned}
& \bar{w}_{, \bar{X} \bar{X}}^{c}-c_{y *}\left(\frac{n_{y *} \pi}{\epsilon}\right)^{2} \nu \bar{w}^{c}=0, \\
& \left(\bar{g}_{*} \bar{w}_{, \bar{X} \bar{X}}^{c}\right)_{, \bar{X}}-c_{y *}\left(\frac{n_{y *} \pi}{\epsilon}\right)^{2}\left(v\left(\bar{g}_{*} \bar{w}^{c}\right)_{, \bar{X}}+2(1-v) \bar{g}_{*} \bar{w}_{, \bar{X}}^{c}\right)=0 .
\end{aligned}
$$

The solution $\bar{w}^{c, F}$ of the buckling equation (13), which satisfies the boundary conditions, Eqs. 21 and 22, can be found by solving the weak form of Eq. 13, which is done by using a combined analytical-numerical solution procedure. In this procedure, $\bar{w}^{c, F}$ is expressed as a linear combination of suitable basis functions that are multiplied by unknown, generalised coordinates defining the corresponding amplitudes. Inserting the expression for $\bar{w}^{c, F}$ into the weak form of Eq. 13 leads to a set of coupled equations, which is solved numerically by using an incremental-iterative (Newton-Raphson) solution procedure. More details about this solution procedure can be found in [21].

As argued in [21], the elastic buckling behaviour of 3D-printed wall structures is uniquely described by the following three dimensionless parameters:

$$
\begin{aligned}
\bar{l}_{c r} & =\lambda^{1 / 3} \kappa=\left(\frac{\rho g h}{D_{0}}\right)^{\frac{1}{3}} l_{c r}, \\
\bar{b} & =\lambda^{1 / 3} \epsilon=\left(\frac{\rho g h}{D_{0}}\right)^{\frac{1}{3}} b, \\
\bar{\xi}_{E} & =\lambda^{-1 / 3}=\left(\frac{D_{0}}{\rho g h}\right)^{\frac{1}{3}} \frac{\xi_{E}}{i}, \quad \text { with } \quad \xi_{E} \in\left\{\xi_{E}^{l}, \xi_{E}^{e}\right\},
\end{aligned}
$$

with $\lambda, \epsilon$ and $\kappa$ given by Eqs. $15_{1}, 15_{2}$ and 20 , respectively. Here, Eq. $23_{1}$ represents the dimensionless critical buckling length, Eq. $23_{2}$ is the dimensionless width of the wall and Eq. 233 reflects the dimensionless "curing rate", which also incorporates the effect by the printing velocity $i$.

In Section 3, the three dimensionless parameters given by Eq. 23 are used for the analysis of the elastic buckling behaviour of the three wall configurations shown in Fig. 2, whereby results calculated by the parametric model are compared to those obtained by FEM simulations. For the free wall configuration, the finite element results are compared against accurate closed-form expressions for the dimensionless bifurcation buckling length, which were established in [21] from numerical results computed with the parametric model. For a free wall characterised by a linear curing process, this closed-form expression reads [21]:

$\bar{l}_{c r}=\bar{l}_{c r, 0}+0.996\left(\bar{\xi}_{E}^{l}\right)^{0.793}$,

with $\bar{l}_{c r}$ and $\bar{\xi}_{E}^{l}$ in accordance with Eqs. $23_{1}$ and $23_{3}$, respectively. The limit value $\bar{l}_{c r, 0}=1.98635$ is the rateindependent dimensionless buckling length of the wall, i.e. the buckling length corresponding to an infinitely fast printing process or, equivalently, to an infinitely slow curing process. For a free wall characterised by an exponentially decaying curing process, the closed-form expression for the buckling length is [21]:

$$
\begin{aligned}
\bar{l}_{c r} & =\bar{l}_{c r, 0}\left[\left(\gamma_{E}\right)^{\frac{1}{3}}+\left(1-\left(\gamma_{E}\right)^{\frac{1}{3}}\right) \exp (-(1.662\right. \\
& \left.\left.\left.+0.240 \gamma_{E}\right) \bar{\xi}_{E}^{e}\right)\right] \quad \text { with } \quad \gamma_{E}=\frac{E_{\infty}}{E_{0}}
\end{aligned}
$$

\subsection{Plastic collapse}

Instead of failing by elastic buckling, during the 3D printing process, the wall structure at its bottom may fail by plastic collapse, as a result of the dead weight stress reaching the material yield strength. Due to the curing process of the printing material, the material yield strength evolves with time. In analogy with Eq. 9, this effect can be formally expressed in terms of the dimensionless Eulerian coordinate $\bar{X}$ as:

$\hat{\sigma}_{p *}(\bar{X})=\hat{\bar{h}}_{*}(\bar{X}) \sigma_{p, 0}$,

in which $\sigma_{p, 0}$ is the initial yield strength (i.e. the yield strength of the material at the moment it leaves the printing nozzle), and $\bar{h}_{*}=\hat{\bar{h}}_{*}(\bar{X})$ reflects the effect of the curing process on the yield strength. Similar to the curing functions (10) and (11) for the elastic stiffness, the two basic curing functions selected for the yield strength are the linear curing function:

$\hat{\bar{h}}_{*}^{l}(\bar{X})=1-\bar{X}, \quad$ with $\quad \bar{X}=\frac{\xi_{\sigma}^{l} X}{i}$,

and the exponentially decaying curing function:

$$
\begin{aligned}
\hat{\bar{h}}_{*}^{e}(\bar{X})= & \gamma_{\sigma}+\left(1-\gamma_{\sigma}\right) \exp (\bar{X}), \\
& \text { with } \quad \bar{X}=\frac{\xi_{\sigma}^{e} X}{i} \quad \text { and } \quad \gamma_{\sigma}=\frac{\sigma_{p, \infty}}{\sigma_{p, 0}} .
\end{aligned}
$$

Here, $\xi_{\sigma} \in\left\{\xi_{\sigma}^{l}, \xi_{\sigma}^{e}\right\}$ are the linear and exponential curing rates, and $\gamma_{\sigma}$ represents the ratio between the final yield strength $\sigma_{p, \infty}$ at the end of the exponentially decaying curing process and the initial yield strength $\sigma_{p, 0}$. As explained in [21], the values of the yield strength $\sigma_{p} \in$ $\left\{\sigma_{p, 0}, \sigma_{p, \infty}\right\}$ depend on the type of failure criterion adopted. A representative criterion is failure by compression, where

$\sigma_{p}=\sigma_{c}$, 
with $\sigma_{c}$ the uniaxial compressive strength. Another representative mechanism is failure by pressure-dependent shear, in accordance with a Mohr-Coulomb type model ${ }^{2}$, i.e.:

$\sigma_{p}=\frac{2 c \cos (\phi)}{1-K_{y}-\left(1+K_{y}\right) \sin (\phi)}$,

in which $c$ and $\phi$ represents the cohesion and the friction angle of the printing material, respectively, and $K_{y}$ is the coefficient of lateral stress, evaluated at the bottom of the wall. For a printing material without frictional resistance $(\phi=0)$, Eq. 30 reduces to:

$\sigma_{p}=\frac{2 c}{1-K_{y}}$,

whereby failure occurs as a result of reaching the maximal shear stress at the bottom of the wall. Which specific criterion for $\sigma_{p}$ is applicable depends on the material characteristics of the actual printing material.

In the case of linear curing, the yield function for plastic collapse can be formulated in a dimensionless form as [21]:

$\bar{l}_{p}=\frac{1}{1-\bar{\xi}_{\sigma}^{l}} \quad$ with $\quad 0 \leq \bar{\xi}_{\sigma}^{l}<1$,

while for exponentially decaying curing it reads [21]:

$\bar{l}_{p}-\left[\gamma_{\sigma}+\left(1-\gamma_{\sigma}\right) \exp \left(-\bar{\xi}_{\sigma}^{e} \bar{l}_{p}\right)\right]=0$.

Here, $\bar{\xi}_{\sigma}$ and $\bar{l}_{p}$ are the dimensionless curing rate and the dimensionless wall length at plastic collapse, respectively, given by:

$\bar{\xi}_{\sigma}=\frac{\xi_{\sigma}\left|\sigma_{p, 0}\right|}{\rho g i} \quad$ with $\quad \xi_{\sigma} \in\left\{\xi_{\sigma}^{l}, \xi_{\sigma}^{e}\right\}$,

$\bar{l}_{p}=\frac{\rho g l_{p}}{\left|\sigma_{p, 0}\right|}$

with $l_{p}$ the actual wall length at plastic collapse. The two dimensionless parameters given by Eq. 34 are sufficient for uniquely describing the plastic collapse behaviour of 3D printed walls. The transcendental equation, Eq. 33, does not have an exact, closed-form solution, and thus needs to be solved numerically, for example, by using a Newton-Raphson solution procedure. Nonetheless, an accurate closed-form

\footnotetext{
${ }^{2}$ Note that the sign of the third term in the denominator of Eq. 30 is opposite from that of the corresponding term in the original expression, Eq. (79), presented in [21]. This is, because in the derivation of Eq. (79) the direction corresponding to the largest absolute value of the principal stress, which is the vertical wall direction, has been erroneously set equal to the horizontal wall direction.
}

approximation for the numerical solution of Eq. 33 is given by [21]:

$\bar{l}_{p}=\bar{l}_{p, 0}\left(1+\frac{\gamma_{\sigma}-1}{1+\left(\frac{\bar{\xi}_{\sigma}^{e}}{\bar{\xi}_{r e f}}\right)^{-p}}\right)$,

with

$$
\bar{\xi}_{\text {ref }}=\hat{\bar{\xi}}_{\text {ref }}\left(\gamma_{\sigma}\right)=\frac{1.181}{1+0.844 \gamma_{\sigma}},
$$

and $\quad p=\hat{p}\left(\gamma_{\sigma}\right)=1.466\left(\gamma_{\sigma}\right)^{0.322}$,

where the initial dimensionless length for plastic collapse equals unity, $\bar{l}_{p, 0}=1$. The closed-form expressions given by Eqs. 32 and 35 are independent of the type of boundary conditions imposed along the vertical wall edges, and therefore are applicable for describing the plastic collapse behaviour of arbitrary straight wall structures.

\subsection{Competition between elastic buckling and plastic collapse}

The wall will fail by yielding if the wall length for plastic collapse is smaller than the critical buckling length, $l_{p}<$ $l_{c r}$. In contrast, when $l_{p}>l_{c r}$, the wall will fail by elastic buckling. This criterion for the determination of the possible failure mechanism can be formulated in terms of geometrical, material and printing process data by making use of Eqs. $23_{1}$ and $34_{2}$, which results into [21]:

$\frac{\bar{l}_{c r}}{\bar{l}_{p}}<\bar{\Lambda}: \quad$ elastic buckling,

$\frac{\bar{l}_{c r}}{\bar{l}_{p}}>\bar{\Lambda}: \quad$ plastic collapse,

with $\quad \bar{\Lambda}=\left(\frac{h}{D_{0}}\right)^{\frac{1}{3}} \frac{\left|\sigma_{p, 0}\right|}{(\rho g)^{\frac{2}{3}}}$,

and $\quad \bar{l}_{c r}=\hat{\bar{l}}_{c r}\left(\bar{\xi}_{E}, \bar{b}\right), \quad \bar{l}_{p}=\hat{\bar{l}}_{p}\left(\bar{\xi}_{\sigma}\right)$.

In summary, Eq. 36 illustrates that the mechanisms of elastic buckling and plastic collapse of a straight 3Dprinted wall can be uniquely described by means of the five dimensionless parameters $\bar{l}_{c r}, \bar{\xi}_{E}, \bar{b}, \bar{l}_{p}$ and $\bar{\xi}_{\sigma}$ given by Eqs. 23 and 34.

\section{Numerical results}

In this section, the failure behaviour during 3D printing is computed for various types of wall structures, among which the basic configurations depicted in Fig. 2, whereby the results calculated with the parametric $3 \mathrm{D}$ printing model are compared against those obtained by FEM simulations. The results from the parametric model relate to specific cases that were included in the extensive parameter variation 
study performed in [21], and thus can be also read off from the corresponding design graphs presented in this reference. The FEM simulations include both a bifurcation analysis and a non-linear buckling analysis for determining the elastic buckling response. The effect of geometrical imperfections on the buckling response is analysed for the free wall configuration. Subsequently, the buckling response of a more advanced, rectangular wall geometry is computed. Finally, for a free wall, the transition of failure by elastic buckling to plastic collapse is demonstrated under a specific change of the material parameters.

In the parametric model, the coefficient of lateral stress is assumed as constant and set equal to Poisson's ratio, $K_{y *}=$ $v$. For the simply supported wall and the fully clamped wall depicted in Fig. 2, this corresponds to a fully constrained, in-plane support along the vertical boundaries (see [21] for more details).

\subsection{FEM modelling}

The FEM simulations of the 3D printing process of wall structures were performed using the commercially available finite element software $\mathrm{Abaqus}^{3}$, and were operated by means of a tailor-made, parametric Phyton script (see also [24]). The wall configurations were modelled as three-dimensional structures, and were constructed in a layer-wise fashion to perform elastic buckling and plastic collapse analyses. The dimensions of the layer were taken representative of those in realistic 3D printing processes. The layers were added stepwisely using the "model change" option in Abaqus.

After the interface with the preceding layer was made coherent by means of geometrical (tie) constraints, the dead weight of the layer was increased linearly with time until it was fully activated. The above process was repeated up to failure of the wall structure by elastic buckling or plastic collapse.

The critical failure modes of the wall configurations depicted in Fig. 2 are symmetric with respect to the vertical symmetry line at the half-width of the wall. Hence, to limit the computational time of the FEM analyses, only one symmetric part of the wall structures was meshed, and roller supports were applied to warrant this symmetry and to prevent rigid body motions. The 3D meshes were constructed using 4-node tetrahedron elements equipped with 1-point Gauss quadrature. Preliminary comparisons with FEM simulations using discrete Kirchhoff quadrilateral shell elements showed to give virtually identical results. Although shell elements are computationally more efficient, tetrahedron elements better facilitate the construction of

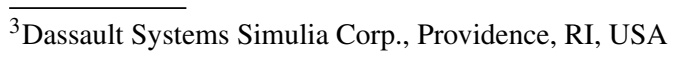

more complex wall geometries, for example when accounting for geometrical imperfections. For this reason, all FEM simulations considered in this communication were performed with 3D tetrahedron elements. The cross-section of each layer was modelled with a total of 32 elements, using two elements across the height of a layer. The total number of elements employed in the simulations depends on the specific geometry modelled, as well as on the critical buckling length of the wall. As an example, for the three basic wall configurations illustrated in Fig. 2, the number of finite elements lies approximately between 65,000 and 135,000 elements for one symmetric part of the wall, which proved to be sufficient to accurately capture the global failure response of the walls. In order to trigger the occurrence of global buckling in the non-linear buckling analysis, an initial imperfection profile corresponding to the first buckling mode-computed from a bifurcation analysis-was applied, whereby the imperfection amplitude was set equal to one-thousandth of the wall thickness. The time-dependent developments of the elastic stiffness and the plastic strength were described by the linear and exponentially decaying curing processes characterised by Eqs. 5 and 6, respectively.

In addition to a non-linear buckling analysis, the wall structures were subjected to an FEM bifurcation buckling analysis. The critical buckling length following from this bifurcation analysis was computed in an iterative fashion, by starting from an initial estimate for the wall length and assigning a stiffness variation in length direction, in accordance with the linear or exponential curing law applied and the printing velocity imposed. Subsequently, by making good use of the scaling of parameters expressed via (23) $)_{1}$, the wall length was adapted based on the computed value of the buckling load factor, until the load factor reached unity within an acceptable inaccuracy of $3 \%$.

\subsection{Elastic buckling}

The printing parameters used in the elastic buckling analyses are listed in Table 1, and are representative of wall structures manufactured by 3D concrete printing [21, 24]. Initially, the three basic configurations sketched in Fig. 2 are considered, which are the free wall, the simply supported wall and the fully clamped wall. The elastic stiffness of the concrete material is characterised by a linear curing process (see Fig. 3), whereby three different dimensionless curing rates are selected that cover a broad range of practical conditions, namely $\bar{\xi}_{E}^{l}=0.02,0.5$ and 2.0 , with the normalised curing rate defined by Eq. $23_{3}$.

Note that a higher normalised curing rate either corresponds to a higher curing rate of the printing material, or to a printing process performed at a lower printing velocity (see Eq. $23_{3}$ ). For the free wall, the dimensionless wall width $\bar{b}$ given by Eq. $23_{2}$ does not influence the 
Table 1 Printing and curing process parameters

\begin{tabular}{ll}
\hline Parameter & Value \\
\hline Wall thickness & $h=43.5(\mathrm{~mm})$ \\
Height of individual layer & $t_{l}=9.2(\mathrm{~mm})$ \\
Material density & $\rho=2020\left(\mathrm{~kg} / \mathrm{m}^{3}\right)$ \\
Velocity of printer head & $v_{n}=83.3(\mathrm{~mm} / \mathrm{s})$ \\
Poisson's ratio & $v=0.3(-)$ \\
$\quad$ Linear curing & \\
Initial elastic stiffness & $E_{0}=0.0781(\mathrm{MPa})$ \\
Curing rate & $\bar{\xi}_{E}^{l}=0.02,0.5,2.0(-)$ \\
$\quad$ Exponential curing & \\
Initial elastic stiffness & $E_{0}=0.0781(\mathrm{MPa})$ \\
Curing rate & $\bar{\xi}_{E}^{e}=0.02,0.5,2.0(-)$ \\
Stiffness ratio & $\gamma_{E}=\frac{E_{\infty}}{E_{0}}=5(-)$ \\
\hline
\end{tabular}

critical buckling length (see Eq. 24), and thus can be given an arbitrary value. For the simply supported and fully clamped walls, the widths were set equal to $\bar{b}=9.1$ and $\bar{b}=13.0$, respectively. The fully clamped wall was given a larger width than the simply supported wall in order to avoid that the buckling length at the higher curing rates selected becomes impractically large, as can be observed from Figure 23 in [21]. The current analyses focus on elastic buckling, whereby the plastic collapse mechanism is excluded by giving the yield strength $\sigma_{p, 0}$ in Eq. 26 a relatively large value. The transition in failure mechanism from elastic buckling to plastic collapse will be addressed in detail in Section 3.5.

Figure 5 shows the buckling response for the three basic wall types at the three curing rates selected. The buckling response is evaluated in terms of the dimensionless critical buckling length $\bar{l}_{c r}$ given by Eq. $23_{1}$. For the free wall, the critical buckling length has been determined from the parametric model by applying the closed-form expression, Eq. 24, while for the simply supported and fully clamped walls it has been computed by solving the weak form of the differential equation, Eq. 13, together with the boundary conditions, Eqs. 21 and 22, using the combined analyticalnumerical solution procedure described in [21].

For the free wall and the simply supported wall, the results from the FEM bifurcation analysis turn out out be in excellent agreement with those from the parametric model: for the three curing rates selected the values calculated for the critical bifurcation buckling length differ less than $2 \%$. For the fully clamped wall, the relative difference is comparably small at the low curing rate $\bar{\xi}_{E}^{l}=0.02$, but tends to grow when the curing rate becomes higher. Specifically, at the largest curing rate of $\bar{\xi}_{E}^{l}=2.0$, the parametric model overestimates the critical buckling length computed by the FEM bifurcation analysis by

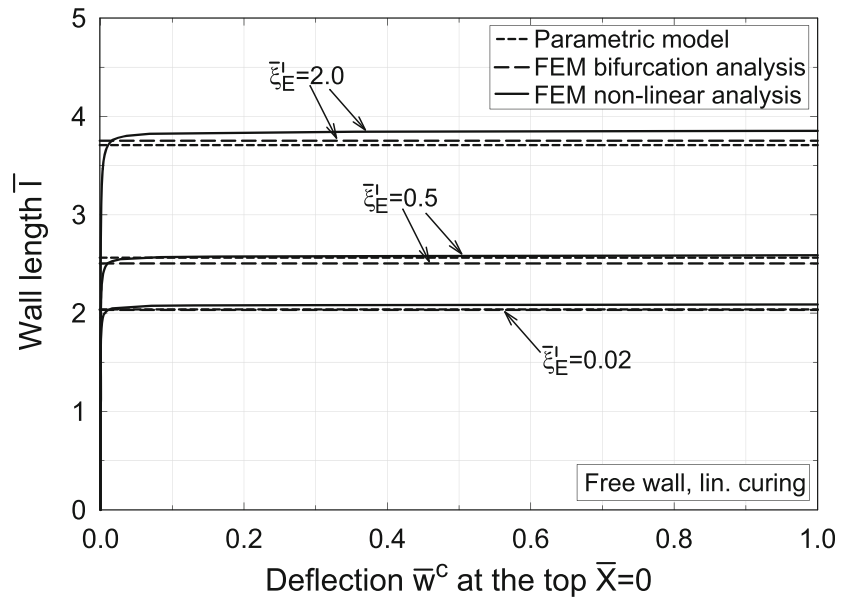

(a) Free wall.

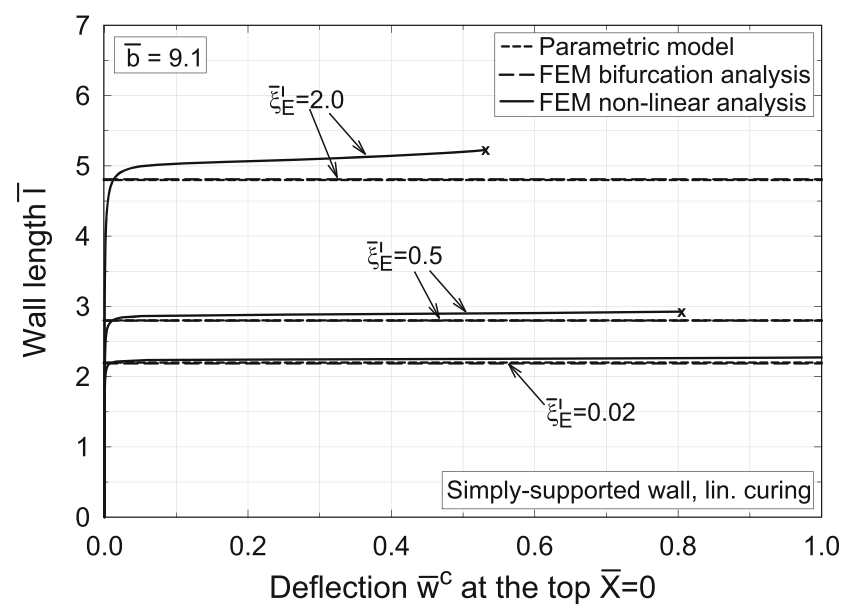

(b) Simply-supported wall.

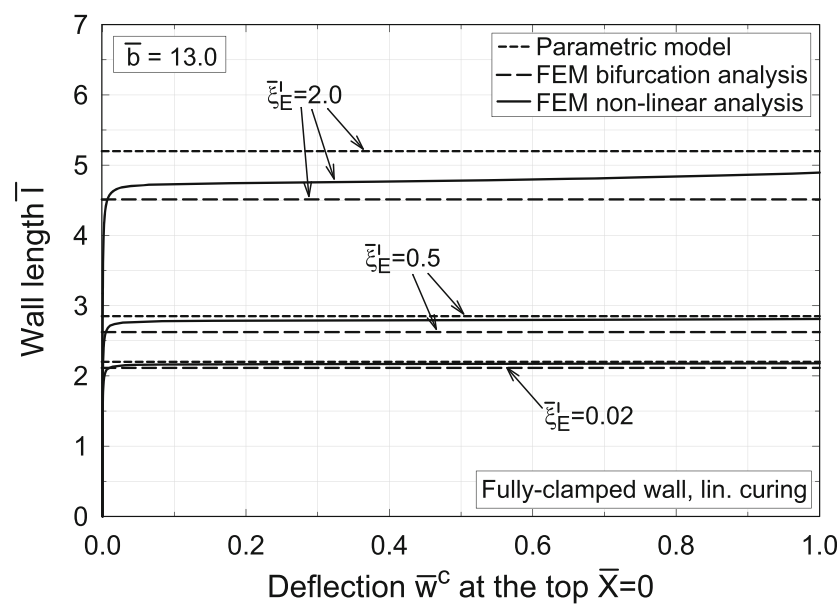

(c) Fully-clamped wall.

Fig. 5 Wall length $\bar{l}$ versus wall top deflection $\bar{w}^{c}$ for a a free wall, b a simply supported wall and $\mathbf{c}$ a fully clamped wall. The walls are subjected to a linear curing process considering three different curing rates, $\bar{\xi}_{E}^{l}=0.02,0.5$ and 2.0. The short-dashed and long-dashed lines indicate the bifurcation buckling lengths obtained from the parametric model and an FEM bifurcation analysis, respectively, and the solid line represents the buckling response from a non-linear FEM analysis 
$15.2 \%$. This difference is due to the cosine approximation, Eq. $22_{3}$, used in the parametric model for describing the horizontal buckling shape of the fully clamped wall. This is illustrated in more detail in Fig. 6, which depicts the cosine approximation together with the horizontal buckling shape calculated by the FEM model for $\bar{\xi}_{E}^{l}=2.0$ along two different layers, namely the 5 th layer (located close to the wall bottom) and the 43rd layer (located at the wall top).

It can be observed that for the 5th layer the approximation given by Eq. $2_{3}$ is rather accurate; only close to the wall boundary there appears to be a noticeable difference with the buckling shape calculated by the FEM model. Conversely, for the 43rd layer, the difference is larger, whereby the horizontal buckling shape assumed for the parametric model near the wall boundary and close to the

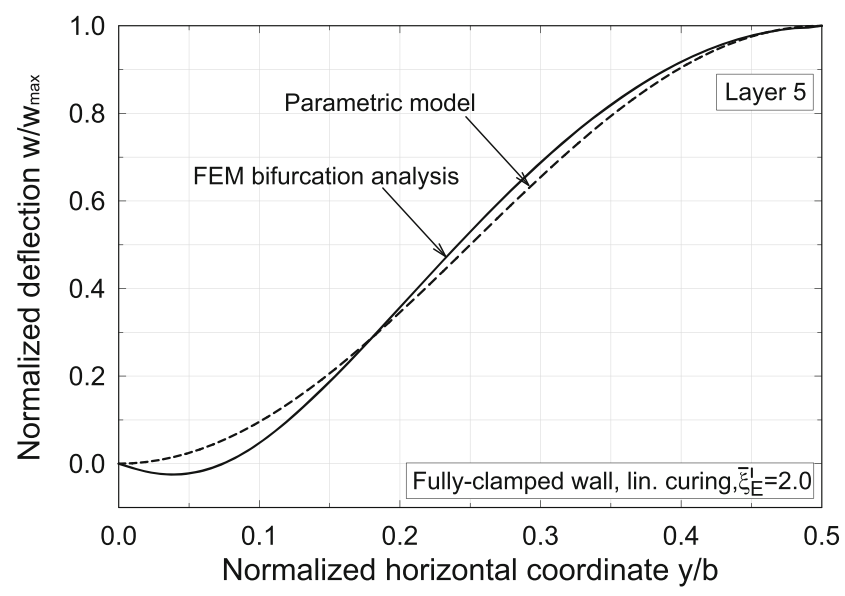

(a) Buckling shape at layer 5 (close to the wall bottom).

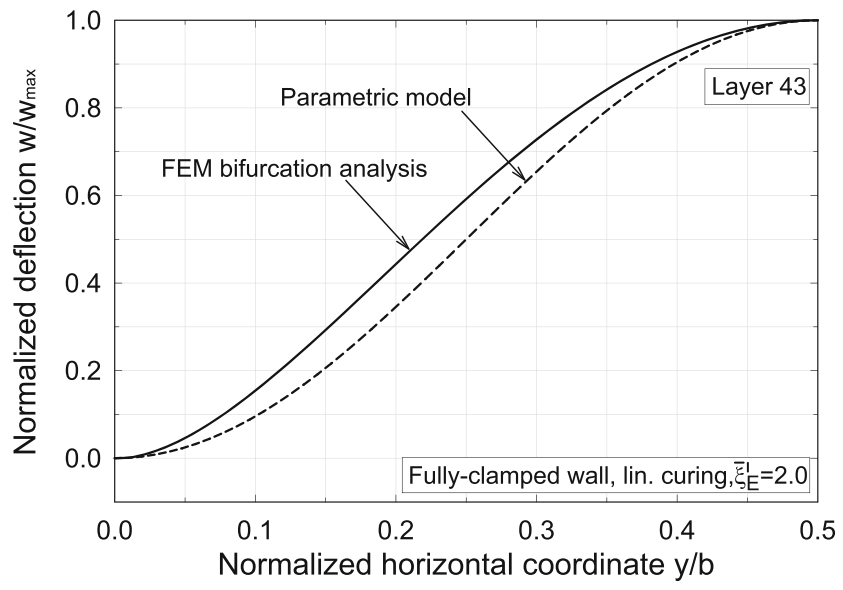

(b) Buckling shape at layer 43 (at the wall top).

Fig. 6 Horizontal buckling shape across the (symmetrical) half-width of a fully clamped wall, evaluated in a layer 5 (close to the wall bottom) and b layer 43 (at the wall top). The wall is subjected to a linear curing process with a relatively high curing rate, $\bar{\xi}_{E}^{l}=2.0$. The short-dashed and solid lines designate the buckling shapes from the parametric model and the FEM bifurcation analysis, respectively half-width is more curved than the shape computed by the FEM model, thus leading to a higher buckling length. Since the discrepancy in horizontal buckling shape becomes stronger with increasing distance from the bottom of the wall, the relative overestimation of the critical buckling length by the parametric model becomes greater at a larger buckling length, as generated under a higher curing rate.

The response following from the non-linear FEM buckling analysis for all three wall configurations lies close to the bifurcation buckling length, especially at the onset of buckling. At larger deformations $\bar{w}^{c}$ the buckling length obtained from the non-linear analysis may show a slight increase, which can be ascribed to a membrane stiffening effect developing in the wall structure. Note from Fig. 5b that for the simply supported wall the non-linear FEM analyses for the curing rates $\bar{\xi}_{E}^{l}=0.5$ and $\bar{\xi}_{E}^{l}=2.0$ at some stage did not converge, as indicated by the small crosses at the end of the buckling response.

In addition to the linear curing process, the free wall is subjected to an exponentially decaying curing process characterised by a stiffness ratio $\gamma_{E}=E_{\infty} / E_{0}=5$, whereby again three different curing rates are considered, $\bar{\xi}_{E}^{e}=0.02,0.5$ and 2.0. The dimensionless critical buckling length $\bar{l}_{c r}$ can be straightforwardly computed using the closed-form expression, Eq. 25, derived in [21] from the numerical results of the parametric model. As illustrated in Fig. 7, the critical buckling lengths calculated with this expression are in excellent agreement with the bifurcation buckling lengths computed with the FEM model, i.e. the relative difference in buckling length lies between 1.0 and $2.6 \%$.

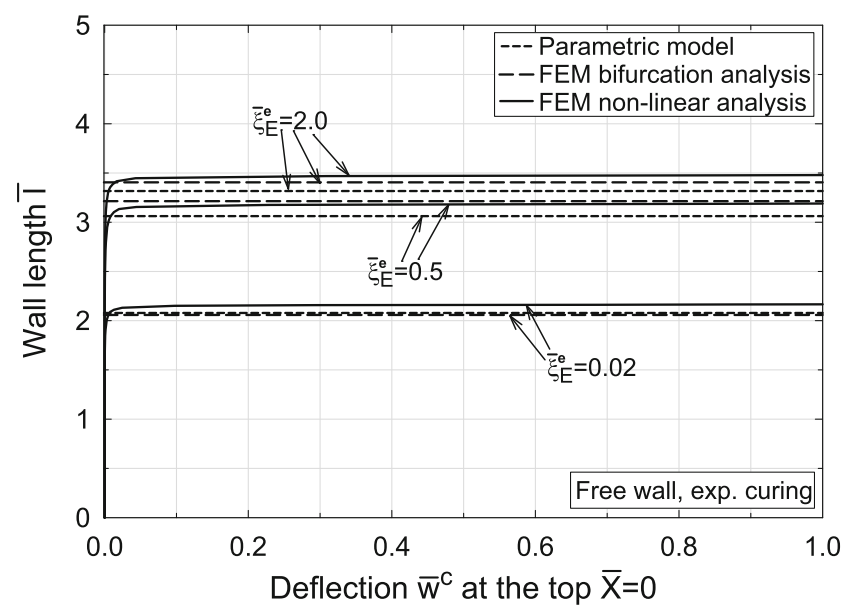

Fig. 7 Wall length $\bar{l}$ versus wall top deflection $\bar{w}^{c}$ for a free wall. The wall is subjected to an exponentially decaying curing process considering three different curing rates, $\bar{\xi}_{E}^{e}=0.02,0.5$ and 2.0. The short-dashed and long-dashed lines indicate the bifurcation buckling lengths obtained from the parametric model and an FEM bifurcation analysis, respectively, and the solid line represents the buckling response from a non-linear FEM analysis 
In addition, the computed bifurcation buckling lengths lie close to the buckling responses following from the non-linear FEM analyses, which illustrates the usefulness of the closed-form expression, Eq. 25, for the design of 3D printing processes. Furthermore, the exponentially decaying trend of the curing function causes the critical buckling length to asymptote to a limit value with increasing curing rate, as illustrated in Fig. 7 by the close correspondence of the critical buckling lengths for the cases $\bar{\xi}_{E}^{e}=0.5$ and 2.0, see [21] for more details on this aspect.

\subsection{Influence of imperfections on buckling response}

The influence of imperfections on the buckling response is evaluated by considering a free wall printed under a linear curing process. For the parametric model, the imperfection profile $\hat{\bar{w}}^{c, 0}(\bar{X})$ present in Eq. 12 is defined by the following kinematically admissible form [21]:

$$
\begin{aligned}
\hat{\bar{w}}^{c, 0}(\bar{X})= & \bar{w}_{m}^{c, 0}\left(-\sin \left(\bar{k}_{w}(\bar{X}+\kappa)\right)\right. \\
& \left.+\bar{\tau}\left[1-\exp \left(-\frac{\bar{k}_{w}}{\bar{\tau}}(\bar{X}+\kappa)\right)\right]\right) .
\end{aligned}
$$

This expression represents a harmonic imperfection, with the exponential term ensuring that the essential boundary conditions given by Eq. 21 are rigorously satisfied. Further, $\bar{w}_{m}^{c, 0}$ is the dimensionless amplitude of the imperfection, $\bar{k}_{w}$ is the dimensionless wavenumber and $\bar{\tau}$ is a boundary factor, in accordance with:

$\bar{k}_{w}=\frac{2 \pi i}{n_{t} t_{l} \xi_{E}}, \quad \bar{\tau}=\frac{2 \pi}{\omega n_{t} t_{l}}$,

Fig. 8 Idealised sinusoidal imperfection profile $w^{c, 0}=\hat{w}^{c, 0}(x)$ used in the parametric model, which is characterised by the amplitude $w_{m}^{c, 0}$ and wavelength $L$, where $L=n_{t} t_{l}$, with $t_{l}$ the height of an individual printed layer and $n_{t}$ the number of layers. The left and right graphs illustrate the cases $n_{t}=2$ and $n_{t}=4$, respectively. The figure has been reprinted from [21] where $t_{l}$ is the height of an individual printed layer, $n_{t}$ is the number of printed layers characterising the wavelength $L$ of the imperfection profile, i.e. $L=n_{t} t_{l}$ (see also Fig. 8) and $\omega$ is a factor defining the influence length of the exponential term at the bottom of the wall.

In the parametric model, the influence length of the exponential term is kept limited by taking a relatively small value for the parameter $\bar{\tau}$ in Eq. 38, i.e. $\bar{\tau}=0.5$. Hence, the imperfection profile given by Eq. 37 becomes fully characterised by the two length-scale parameters $\bar{k}_{w}$ and $\bar{w}_{m}^{c, 0}$. Note that the dimensionless wavenumber may be converted into the dimensionless wavelength of the imperfection profile via:

$\bar{L}=\frac{2 \pi}{\bar{k}_{w}}=\frac{n_{l} t_{l} \xi_{E}}{i}$.

The effect of wall imperfections on the buckling response is analysed by taking the dimensionless imperfection amplitude as $\bar{w}_{m}^{c, 0}=0.05$, and the dimensionless curing rate as $\bar{\xi}_{E}^{l}=2.0$. Three different profiles are selected, whereby the wavenumbers are $\bar{k}_{w}=1,2$ and 20, which correspond to wavelengths $\bar{L}=6.28,3.14$ and 0.314 , respectively, in accordance with Eq. 39. These profiles were studied previously in [21] using the parametric 3D printing model.

In contrast to the parametric model, in which the imperfection profile is treated as a continuous function, see Eq. 37, in the FEM model the displacement calculated from the imperfection profile at the half-height of each layer is applied uniformly across the layer height. In other words, in the FEM model, each layer is subjected to a specific horizontal shift. Figure 9a and b respectively show the buckling responses calculated with the parametric model and the FEM model, by plotting the dimensionless wall length $\bar{l}$
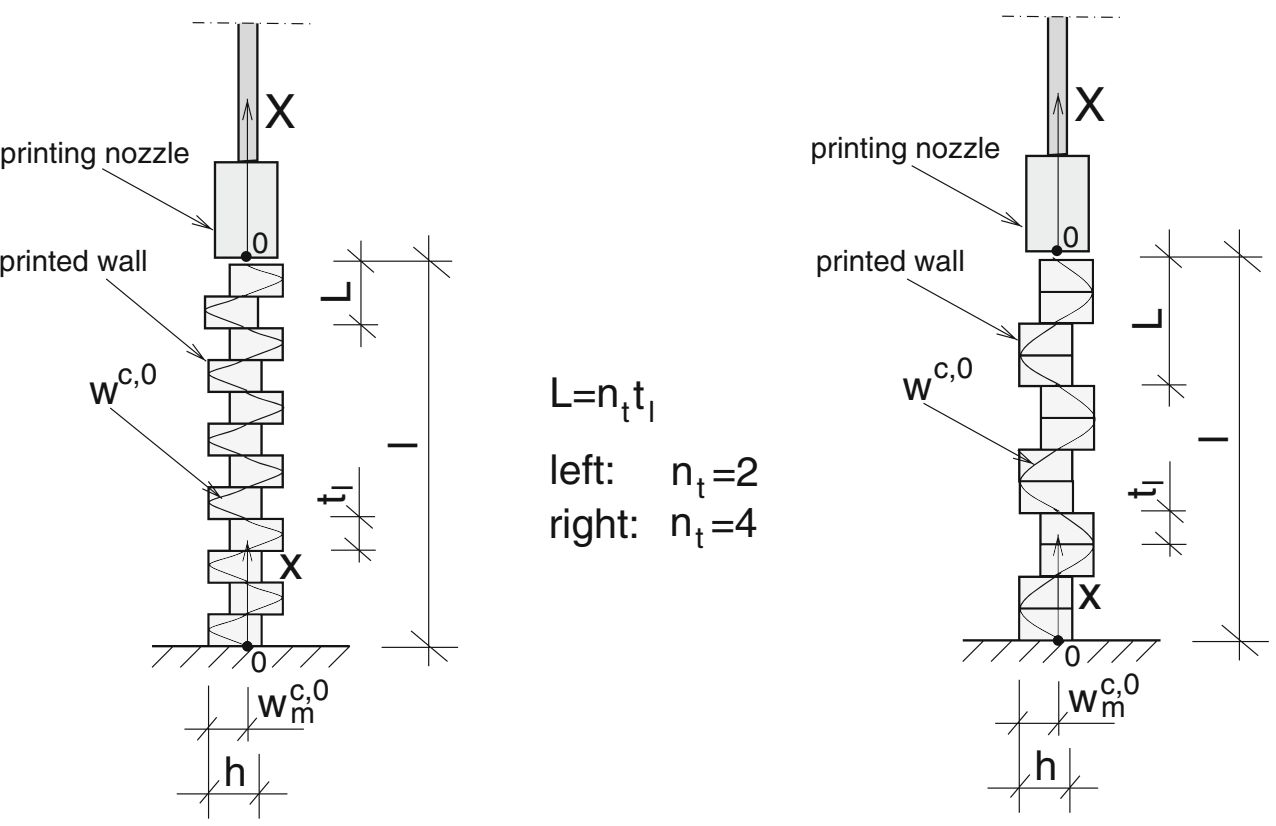


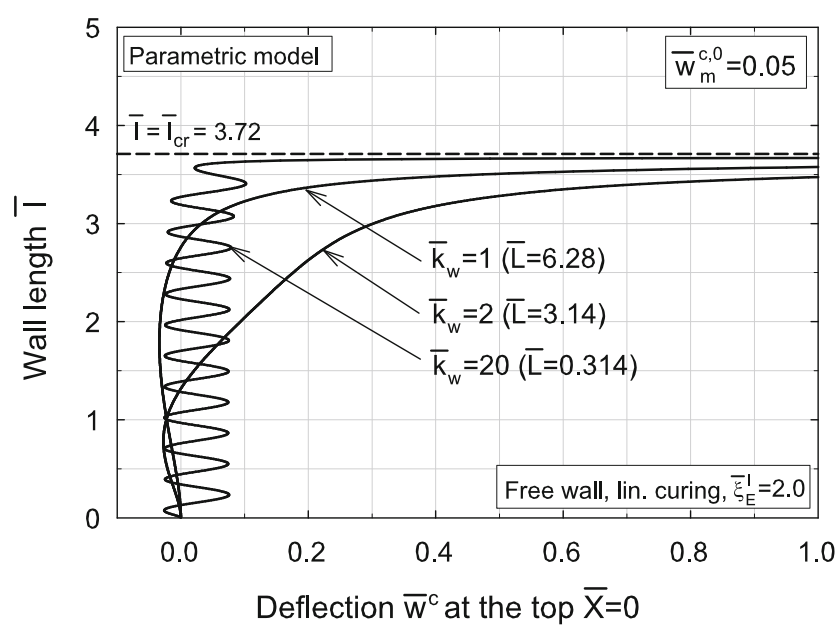

(a) Buckling response from the parametric model.

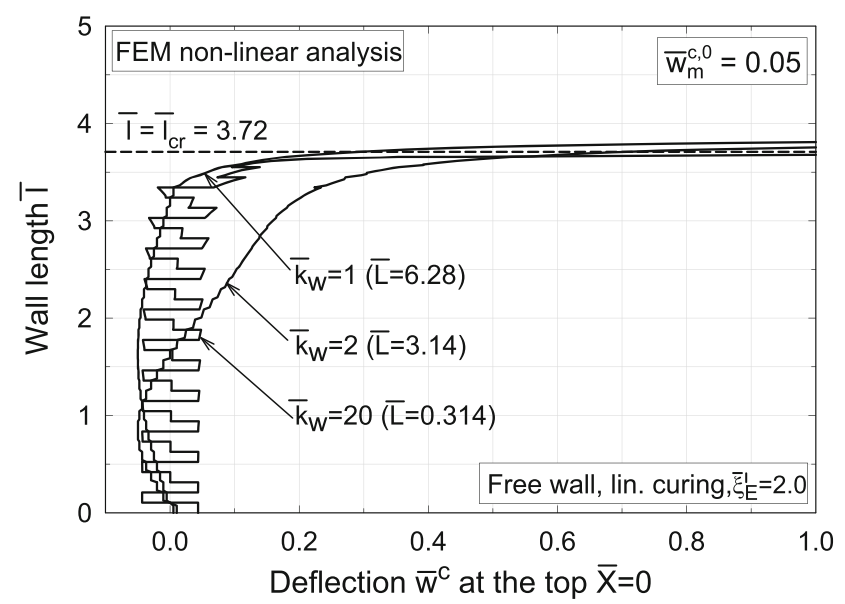

(b) Buckling response from a non-linear FEM analysis.

Fig. 9 Wall length $\bar{l}$ versus wall top deflection $\bar{w}^{c}$ for a free wall with geometrical imperfections, with a the buckling response from the parametric model, and $\mathbf{b}$ the buckling response from a non-linear FEM analysis. The short-dashed line indicates the corresponding bifurcation buckling length. The imperfection amplitude is $\bar{w}_{m}^{c, 0}=0.05$, and the wavenumbers (wavelengths) of the imperfection profiles considered are $\bar{k}_{w}=1,2$ and $20(\bar{L}=6.28,3.14$ and 0.314$)$. The free wall is subjected to a linear curing process at a relatively high curing rate, $\bar{\xi}_{E}^{l}=2.0$. Figure $9 \mathrm{a}$ has been reprinted from [21]

as a function of the dimensionless horizontal deflection $\bar{w}^{c}$ at the wall top, $\bar{X}=0$. Clearly, the results of the two models are comparable, and show that the responses computed for the different wavenumbers at growing horizontal deflection all approach the corresponding bifurcation buckling length (taken from Fig. 5a). For the intermediate wavenumber $\bar{k}_{w}=2$ (corresponding to $\bar{L}=3.14$ ), the critical bifurcation length is reached at a relatively large wall top displacement, while for the smallest and largest wavenumbers, $\bar{k}_{w}=1(\bar{L}=6.28)$ and $k_{w}=20(\bar{L}=$ $0.314)$, the horizontal displacement only starts to grow when the wall length $\bar{l}$ is already relatively close to the bifurcation buckling length $\bar{l}_{c r}$. As argued in [21], the stage at which the horizontal displacement starts to grow is determined by the specific interplay between the two length-scales $\bar{L}$ and $\bar{l}_{c r}$.

\subsection{Buckling of a rectangular wall structure}

Consider now a wall structure with a rectangular layout, composed of two primary walls of width $b$ and two secondary, supporting walls of width $d$, whereby the thickness of the walls is $h$ and the bending stiffness is $D_{*}$ (see Fig. 10). The rectangular wall structure is subjected to a linear curing process, with the values for the curing rate selected as $\bar{\xi}_{E}^{l}=0.02,0.5$ and 1.5 . The wall widths of the primary and secondary walls are $b=800 \mathrm{~mm}$ and $d=300 \mathrm{~mm}$. The values of the other parameters characterising the printing process are listed in Table 1. In accordance with Eqs. 18 and 19, the computation of the critical buckling length of this structure starts by calculating an expression for the dimensionless rotational stiffness $\bar{k}_{r}=\hat{\bar{k}}_{r}(\bar{X})$ to determine the number of half-waves $n_{y *}$ and the constraint factor $c_{y *}$. As discussed in [21], the rotational stiffness can be conveniently formulated in a dimensionless form as:

$\bar{k}_{r}=\hat{\bar{k}}_{r}(\bar{X})=\frac{b \hat{k}_{r}(\bar{X})}{D_{*}}=\frac{b \hat{k}_{r}(\bar{X})}{D_{0} \hat{\bar{g}}_{*}(\bar{X})}$,

with the initial bending stiffness $D_{0}$ given by Eq. 16 and the curing function $\hat{\bar{g}}_{*}(\bar{X})$ presented by Eq. 10 . For the rectangular wall layout, the rotational stiffness, Eq. 40 , characterises the rotational constraint imposed by a supporting wall $d$ on a primary wall $b$, which, for a linear curing process, can expressed as [21]:

$\bar{k}_{r}=\hat{\bar{k}}_{r}(\bar{X})=\frac{2 b D_{0}^{s}}{d D_{0}} \frac{1}{\hat{F}(\bar{X})} \quad$ with $\quad-\kappa \leq \bar{X} \leq 0$,

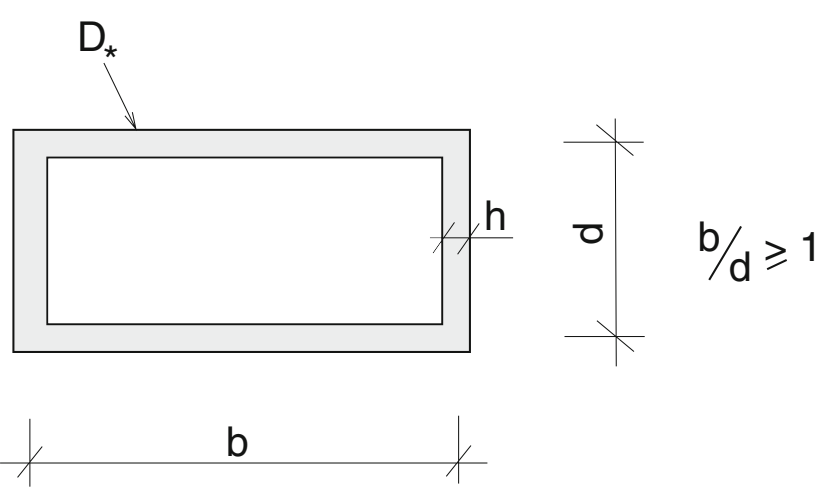

Fig. 10 Rectangular wall-layout composed of two primary walls of width $b$ and two secondary, supporting walls of width $d$. The thickness of the walls is $h$, and the bending stiffness is given by $D_{*}$ 
with

$$
\begin{aligned}
\hat{F}(\bar{X})= & {[4 \sin (\psi) \cos (\psi)(\exp (\psi)+\exp (-\psi))} \\
& +2 \cos (\psi)(\exp (2 \psi)-\exp (-2 \psi)) \\
& -2 \sin (\psi)(\exp (2 \psi)+\exp (-2 \psi))-4 \sin (\psi) \\
& -\exp (3 \psi)+\exp (-3 \psi)-\exp (\psi)+\exp (-\psi)] \\
& \times\left[\psi \left(4 \cos ^{2}(\psi)(\exp (\psi)+\exp (-\psi))\right.\right. \\
& \quad-\exp (3 \psi)-\exp (-3 \psi)-3(\exp (\psi)+\exp (-\psi)))]^{-1},
\end{aligned}
$$

whereby:

$$
\begin{gathered}
\psi=\hat{\psi}(\bar{X})=\left[\frac{30}{4}\left(\bar{X}^{2}-12 \bar{X} \kappa+15 \kappa^{2}-14 \bar{X}+42 \kappa+28\right)(\bar{X}-1)\right]^{1 / 4} \\
\times\left[-73 \bar{X}^{5}-15 \bar{X}^{4} \kappa+390 \bar{X}^{3} \kappa^{2}+530 \bar{X}^{2} \kappa^{3}+195 \bar{X} \kappa^{4}-3 \kappa^{5}\right. \\
+350 \bar{X}^{4}+840 \bar{X}^{3} \kappa+420 \bar{X}^{2} \kappa^{2}-280 \bar{X} \kappa^{3}-210 \kappa^{4} \\
\left.\quad-280 \bar{X}^{3}-840 \bar{X}^{2} \kappa-840 \kappa^{2} \bar{X}-280 \kappa^{3}\right]^{-1 / 4}
\end{gathered}
$$

It can be confirmed that the function $\hat{F}(\bar{X})$ is 0 at the clamped support $\bar{X}=-\kappa$, which, in accordance with Eq. 41, indeed results in $\bar{k}_{r} \rightarrow \infty$. Inserting the above expression for $\bar{k}_{r}$ into Eq. 19 leads to the corresponding functions $\hat{n}_{y *}(\bar{X})$ and $\hat{c}_{y *}(\bar{X})$, which in turn are substituted into the equilibrium equation, Eq. 13, and the natural boundary conditions, Eq. 22, to solve for the buckling response of the rectangular wall geometry (see [21] for more details)

Figure 11 1shows the dimensionless wall length $\bar{l}$ as a function of the dimensionless deflection $\bar{w}^{c}$ evaluated at the top of the wall, adopting a linear curing process and selecting three different dimensionless curing rates, $\bar{\xi}_{E}^{l}=0.02,0.5$ and 1.5. The FEM results are computed for one symmetrical quarter of the rectangular wall layout, using meshes in between 240,000 and 566,000 tetrahedron elements equipped with a 1-point Gauss quadrature. For all the three curing rates considered, the results from the parametric model and the FEM bifurcation analysis are in excellent agreement, with the relative difference varying between $0.5 \%$ (for $\bar{\xi}_{E}^{l}=0.5$ ) and $2.4 \%$ (for $\bar{\xi}_{E}^{l}=$ 0.02). The non-linear buckling analysis approaches the critical buckling length under increasing wall deflection, and subsequently crosses it due to a membrane-stiffening effect in the wall structure. The membrane-stiffening effect becomes stronger under a higher curing rate and, as

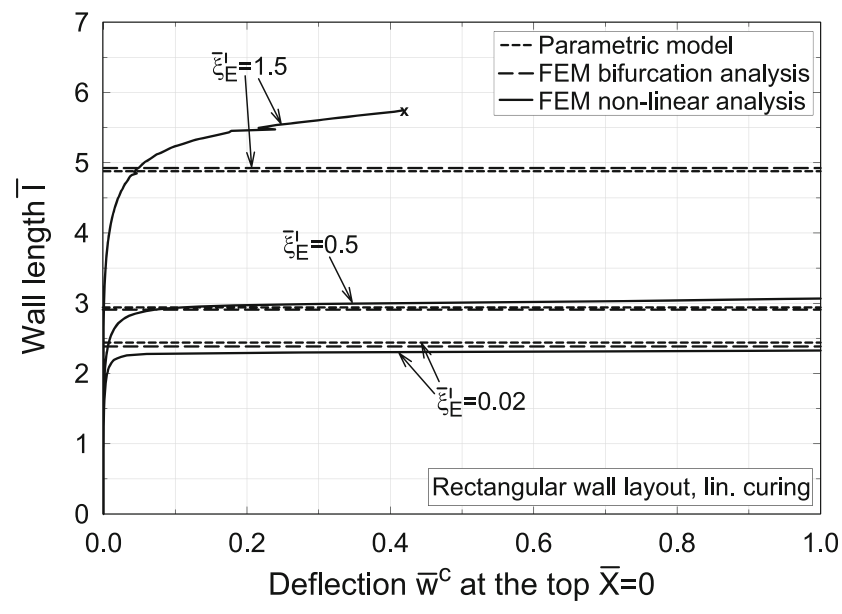

Fig. 11 Wall length $\bar{l}$ versus wall top deflection $\bar{w}^{c}$ for a rectangular wall layout with ground plane dimensions $b \times d=800 \times 300 \mathrm{~mm}^{2}$. The wall is subjected to a linear curing process at curing rates $\bar{\xi}_{E}^{l}=$ $0.02,0.5$ and 1.5. The short-dashed and long-dashed lines indicate the bifurcation buckling lengths obtained from the parametric model and an FEM bifurcation analysis, respectively, and the solid line represents the buckling response from a non-linear FEM analysis

illustrated for $\bar{\xi}_{E}^{l}=1.5$, at some stage may lead to a substantially larger wall length than the critical wall length following from the bifurcation analysis. The buckling shape calculated from the FEM bifurcation analysis for $\bar{\xi}_{E}^{l}=$ 0.5 is shown in Fig. 12, with the colours representing the relative magnitude of the local out-of-plane deflection. For this symmetric buckling shape, the maximal deflection of the primary wall $b$ indeed is substantially larger than that of the secondary, supporting wall $d$, namely a factor of 6.6.

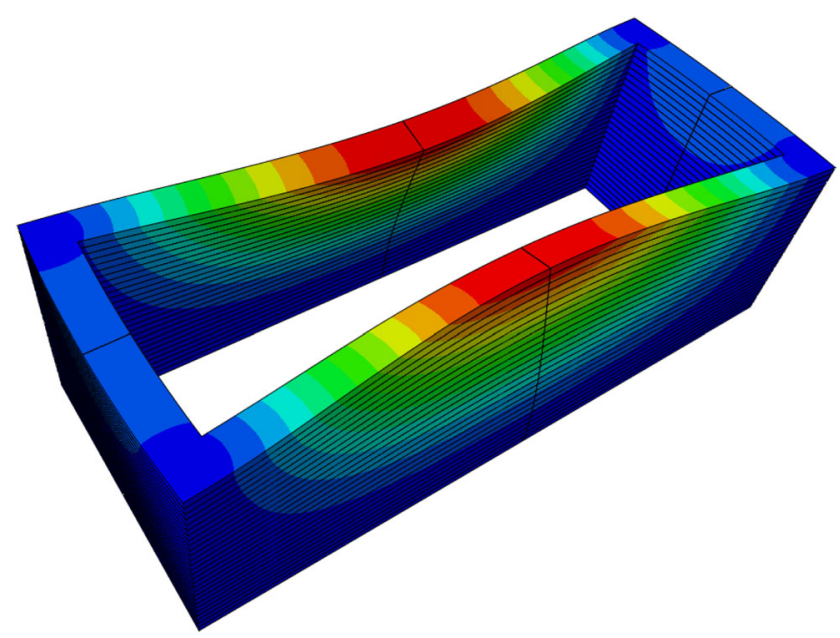

Fig. 12 Deflection profile computed from a FEM bifurcation analysis for a rectangular wall layout subjected to linear curing at a moderate curing rate, $\bar{\xi}_{E}^{l}=0.5$. The colours represent the relative magnitude of the local out-of-plane displacement, with the maximum displacement of the primary wall that buckles being a factor of 6.6 larger than that of the secondary, supporting wall 


\subsection{Plastic collapse}

The transition from failure by elastic buckling to plastic collapse can be clearly demonstrated via a specific selection of the properties of the printing material. Plastic collapse is assumed to occur in accordance with the Mohr-Coulomb failure criterion, with the values for the initial cohesion $c_{0}$ and the initial friction angle $\phi_{0}$ presented in Table 2. These strength values are representative of fresh concrete used in $3 \mathrm{D}$ printing processes [33]. During 3D printing processes, failure by plastic collapse typically takes place at the bottom of the wall, at which the stress generated under dead weight loading is maximal and at a certain stage reaches the material yield strength. In the analysis, the bottom layer is considered to be unconstrained in the longitudinal direction, which is representative of a layer deposited on an ideally smooth surface. In the Mohr-Coulomb failure criterion given by Eq. 30, this corresponds to a coefficient of lateral stress $K_{y}=0$. The simplification made here for the unknown and complicated surface conditions in the $3 \mathrm{D}$ printing process is reasonable, since the present purpose is to compare the results of the FEM model to those of the parametric model by means of a basic, illustrative example, rather than to simulate the surface conditions as realistic as possible.

The transition from failure by elastic buckling to failure by plastic collapse will be illustrated by considering two cases characterised by a linear curing process, which are specified in Table 2 as Case 1 and Case 2. The two cases differ by the choice of the curing rate for the yield strength, which are $\bar{\xi}_{\sigma}^{l}=0.4$ (Case 1) and $\bar{\xi}_{\sigma}^{l}=0.5$ (Case 2). The specific failure mechanism that will occur is set by the criterion in Eq. 36, for which the parameters are determined as follows: Substituting the values for $\phi_{0}$ and $c_{0}$ listed in Table 2, together with $K_{y}=0$, into the Mohr-Coulomb model, Eq. 30, leads to an initial yield strength of $\sigma_{p, 0}=$ $3.48 \mathrm{kPa}$. In accordance with the data presented in Table 1, the initial bending stiffness follows from Eq. 16 as $D_{0}=$ $0.589 \mathrm{Nm}$. Inserting the values for the initial yield strength

Table 2 Material and printing parameters used for indicating the transition from failure by elastic buckling (Case 1) to plastic collapse (Case 2)

\begin{tabular}{ll}
\hline Parameter & Value \\
\hline Initial friction angle & $\phi_{0}=20^{\circ}$ \\
Initial cohesion & $c_{0}=1.22(\mathrm{kPa})$ \\
$\quad$ Case 1 & \\
Curing rate elastic stiffness & $\bar{\xi}_{E}^{l}=2.0(-)$ \\
Curing rate yield strength & $\bar{\xi}_{\sigma}^{l}=0.4(-)$ \\
$\quad$ Case 2 & \\
Curing rate elastic stiffness & $\bar{\xi}_{E}^{l}=2.0(-)$ \\
Curing rate yield strength & $\bar{\xi}_{\sigma}^{l}=0.5(-)$ \\
\hline
\end{tabular}

and the initial bending stiffness, and the values for $\rho$ and $h$ provided in Table 1 into Eq. 36 leads to $\Lambda=1.99$. Subsequently, the critical buckling length of the free wall is calculated with Eq. 24, which, with the value for $\bar{\xi}_{E}^{l}$ provided in Table 2, results in a dimensionless buckling length of $\bar{l}_{c r}=3.71$. The dimensionless length for plastic collapse is obtained from Eq. 32, and, using the curing rates for the yield strength listed in Table 2, equals $\bar{l}_{p}=1.67$ for Case 1 and $\bar{l}_{p}=2.00$ for Case 2. Correspondingly, for Case 1 , the ratio $\bar{l}_{c r} / \bar{l}_{p}=3.71 / 1.67=2.22$, which is larger than $\Lambda=1.99$, so that in accordance with Eq. 36 it may be concluded that the wall will fail by plastic collapse. For Case 2 , the ratio $\bar{l}_{c r} / \bar{l}_{p}=3.71 / 2.00=1.86$, which is smaller than $\Lambda=1.99$, as a result of which Eq. 36 dictates that the wall will fail by elastic buckling. The two different failure mechanisms were indeed confirmed by the FEM simulations, whereby the critical failure lengths for plastic collapse (Case 1) and elastic buckling (Case 2) only differ by, respectively, $0.01 \%$ and $1.20 \%$ from the above values calculated with the parametric model. For a more general consideration of the competition between elastic buckling and plastic collapse of the free wall, the reader is referred to [21], in which the parametric 3D printing model has been used to construct failure mechanism maps for a wide range of printing process parameters.

\section{Experimental validation}

In order to illustrate the usefulness of the parametric model in predicting and understanding the structural failure behaviour in real $3 \mathrm{D}$ printing processes, a series of experiments was performed whereby free wall structures were built layer-bylayer using extrusion-based 3D concrete printing. The experiments were carried out with the 3D concrete printing facility at the Eindhoven University of Technology (see Fig. 13).

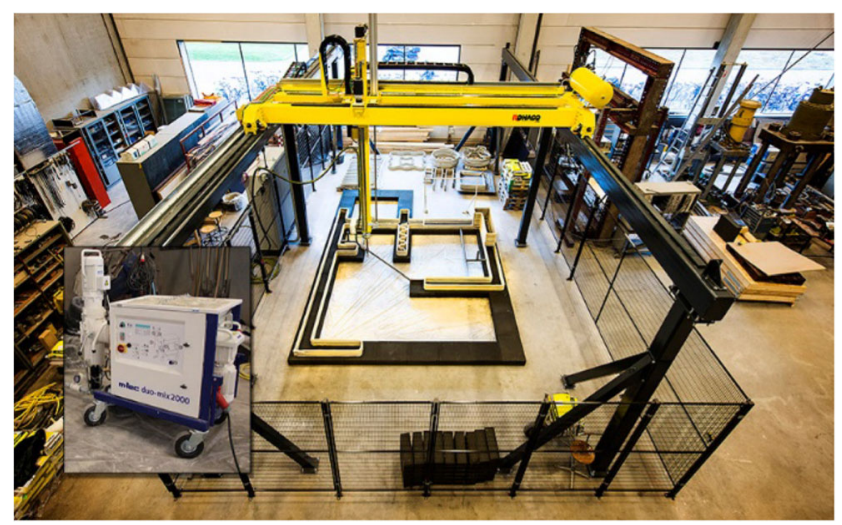

Fig. 13 3D concrete printing facility at the Eindhoven University of Technology, including a 4-axis gantry robot, a control unit and a concrete mixer and pump (inset). The figure has been reprinted from [24] 
Table 3 Printing process parameters for the free wall

\begin{tabular}{ll}
\hline Parameter & Value \\
\hline Wall widths & $b=1.0,5.0,10.4(\mathrm{~m})$ \\
Wall thickness & $h=60.0(\mathrm{~mm})$ \\
Height of individual layer & $t_{l}=9.5(\mathrm{~mm})$ \\
Concrete density & $\rho=2100\left(\mathrm{~kg} / \mathrm{m}^{3}\right)$ \\
Velocity of printer head & $v_{n}=6250(\mathrm{~mm} / \mathrm{min})$ \\
\hline
\end{tabular}

The walls were printed at room temperature, and the effect of the dimensionless curing rate on the failure response was examined by considering three different widths, namely $b=1.0 \mathrm{~m}, 5.0 \mathrm{~m}$ and $10.4 \mathrm{~m}$ (see Table 3 for an overview of these and other process parameters). From initial predictions made with the parametric 3D printing model, it has been a priori estimated that with these process parameters plastic collapse does not become critical, so that the three free walls tested all may be assumed to fail by elastic buckling. As argued in [21], the geometrical accuracy of the printing process is sufficiently high to ignore the effect of imperfections on the value of the critical buckling length. Note hereby that, at a given printing velocity, a wall of a larger width allows the individual layers to have more time to cure during the printing process, which is expected to lead to a higher critical buckling length.

The custom-designed concrete (Weber 3D 145-2) used in the experiments is composed of Portland cement (CEM I $52.5 \mathrm{R}$ ), a siliceous aggregate with a maximum particle size of $1 \mathrm{~mm}$, limestone filler, rheology modifiers, additives and a small quantity of polypropylene fibres [33]. The composition was mixed with water into a homogeneous viscous substance, and subsequently pumped via a hose towards the printer head, at which it was discharged from the printing nozzle to form a layer. The calculated path followed by the printer head was conducted by a motioncontrolled gantry robot with 4 degrees of freedom, i.e. 3 mutually perpendicular translations and 1 rotation about the vertical axis. The setting of appropriate process parameters, such as the concrete viscosity, the printing velocity, the pump pressure, the printing rotation angle, the height of the printer head above the printed layer and the properties of the nozzle opening, was achieved by means of an extensive test program (see [20] for more details).

\subsection{Results}

In accordance with the process parameters listed in Table 3, the volume of fresh concrete discharged per unit time can be calculated as $Q=v_{n} h t_{l}=59375 \mathrm{~mm}^{3} / \mathrm{s}$, and the period for the printing of an individual layer equals $T_{l}=b / v_{n}=9.6$ $\mathrm{s}, 48.0 \mathrm{~s}$ and $99.8 \mathrm{~s}$ for the walls of $1.0 \mathrm{~m}, 5.0 \mathrm{~m}$ and 10.4 $\mathrm{m}$ width, respectively. Substituting these values into Eq. 3 leads to corresponding wall growth velocities of $i=0.990$ $\mathrm{mm} / \mathrm{s}, 0.198 \mathrm{~mm} / \mathrm{s}$ and $0.095 \mathrm{~mm} / \mathrm{s}$, respectively.

The evolution of the stiffness properties of the concrete was measured by performing uniaxial compression tests in accordance with the ASTM D1266 [34]. As described in detail in [33], specimens were prepared at four different curing times, i.e. 15, 30, 60 and $90 \mathrm{~min}$, and were loaded in a displacement-controlled fashion in an Instron test rig by applying a loading rate of $15 \mathrm{~mm} / \mathrm{min}$. At each curing level, five to six different specimens were tested to take into account the statistical spread in material properties. The relation between the elastic stiffness (in $\mathrm{kPa}$ ) and curing time (in min) has been established by applying a leastsquares procedure to the experimental data (see Fig. 14). Accepting a linear best fit with $R^{2}=0.96$ on the average values of the measured stiffness moduli gives the following relation:

$$
\begin{aligned}
& \hat{E}_{*}(t)=39.5+1.705 t \\
& \text { with } \quad E_{*} \text { in } \mathrm{kPa} \text { and } t \text { in } \min .
\end{aligned}
$$

Comparing the above relation to the expressions for linear curing given by Eqs. 4 and 5, the initial stiffness modulus follows as $E_{0}=39.5 \mathrm{kPa}$ and the linear curing rate of the stiffness becomes $\xi_{E}^{l}=1.705 / 39.5=0.0432 \mathrm{~min}^{-1}$ $=7.2 \times 10^{-4} \mathrm{~s}^{-1}$. Adopting Poisson's ratio of $v=0.3$, which is a realistic value for fresh concrete used in 3D printing processes $[21,24,33]$, from Eq. 16 the initial wall bending stiffness can be calculated as $D_{0}=0.781 \mathrm{Nm}$. With Eq. $23_{3}$, the dimensionless curing rates related to the free walls with widths $b=1.0 \mathrm{~m}, 5.0 \mathrm{~m}$ and $10.4 \mathrm{~m}$ become $\bar{\xi}_{E}^{l}=0.062,0.312$ and 0.650 , respectively. In the $3 \mathrm{D}$ printing experiments, the walls with $b=1.0 \mathrm{~m}, 5.0$

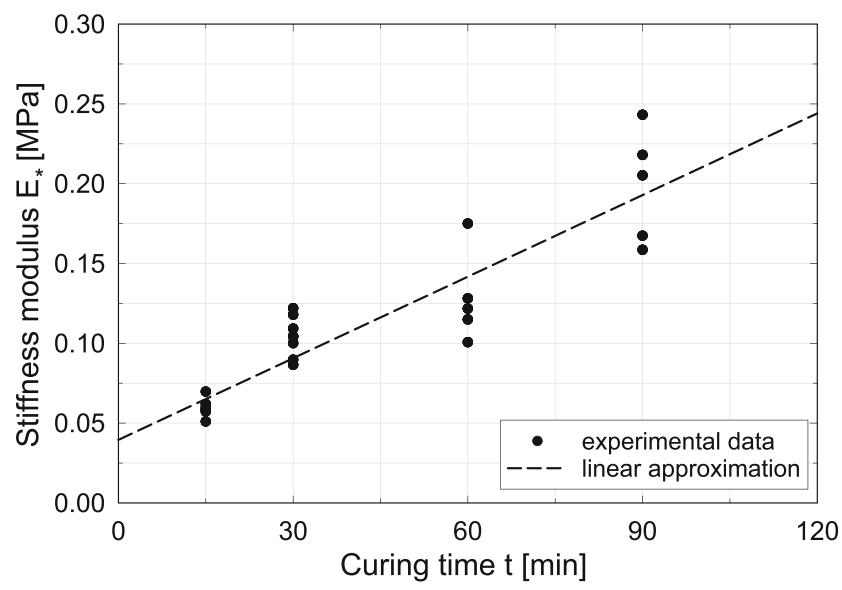

Fig. 14 Elastic stiffness $E_{*}$ measured in uniaxial compression tests (black dots) at 4 different curing times, i.e. 15, 30, 60 and 90 min, together with the linear approximation, Eq. 44, of the average values of the test data (dashed line). The relative standard deviation of $E_{*}$ fluctuates between 10 and $22 \%$ within the range of considered curing times 
$\mathrm{m}$ and $10.4 \mathrm{~m}$ buckled after printing 21, 27 and 46 layers, respectively. Figure 15 depicts the buckling response of the wall with width $b=1.0 \mathrm{~m}$, showing the process from buckling initiation towards full collapse during the printing of the final layer. The buckling responses for the walls with widths $5.0 \mathrm{~m}$ and $10.4 \mathrm{~m}$ are comparable, and are omitted here for brevity. With a layer height of $t_{l}=9.5 \mathrm{~mm}$, the above-mentioned number of layers at wall buckling for $b=$ $1.0 \mathrm{~m}, 5.0 \mathrm{~m}$ and $10.4 \mathrm{~m}$ turn into buckling lengths of $l_{c r}=$ $199.5 \mathrm{~mm}, 256.5 \mathrm{~mm}$ and $437.0 \mathrm{~mm}$, respectively, and, with Eq. $23_{3}$, to dimensionless buckling lengths of $\bar{l}_{c r}=$ 2.32, 2.99 and 5.09. In Fig. 16, these three experimental values for the dimensionless buckling length are plotted versus the corresponding dimensionless curing rates (black dots), together with predictions from the parametric model (black lines) using a linear curing function, Eq. 5, and a

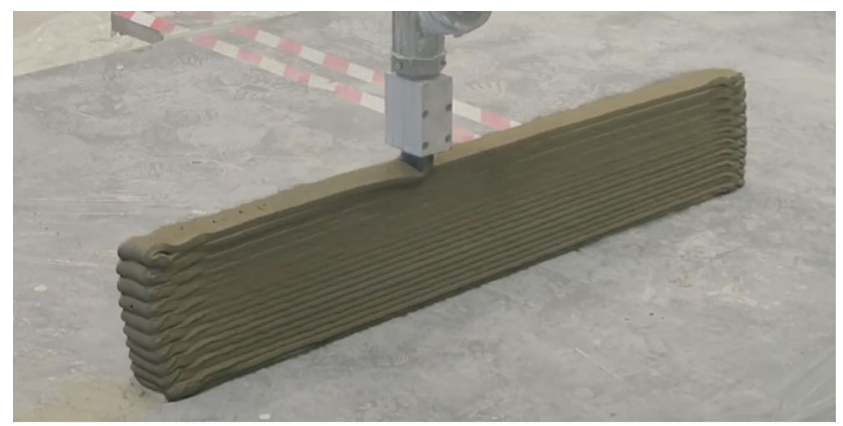

(a) Buckling initiation.

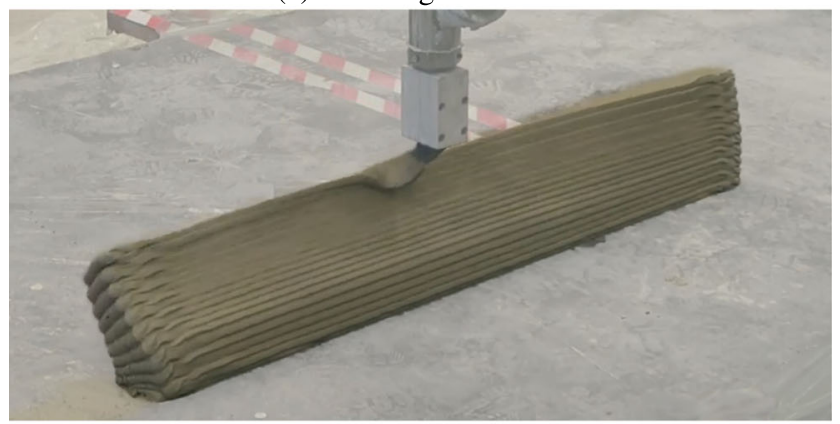

(b) Buckling development.

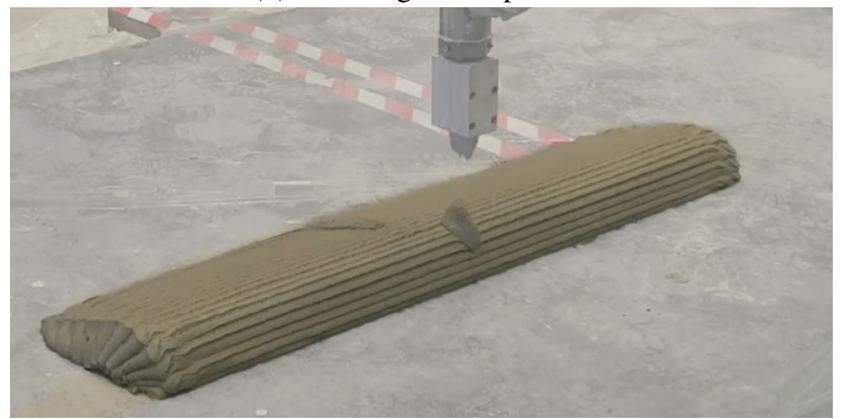

(c) Full collapse.

Fig. 15 Experimental buckling response of the free wall with width $b=1.0 \mathrm{~m}$ at three stages of failure: a buckling initiation, $\mathbf{b}$ buckling development and $\mathbf{c}$ full collapse

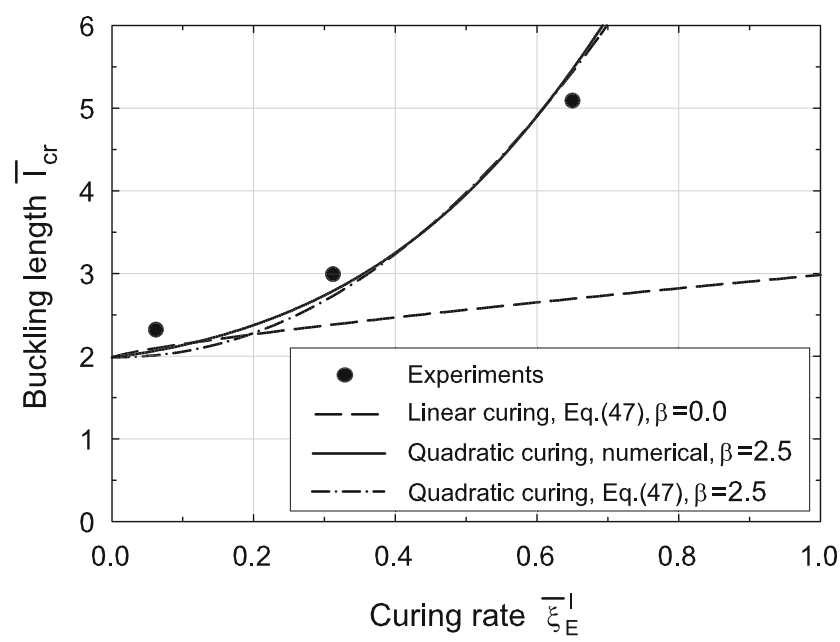

Fig. 16 Dimensionless critical buckling length $\bar{l}_{c r}$ versus the dimensionless curing rate $\bar{\xi}_{E}^{l}$ for a free wall, illustrating experimental results (black dots) and the results from the parametric model (black lines). The curve related to the linear curing function follows from Eq. 47 with $\beta=0.0$ (dashed line), and the curves related to the quadratic curing function follow from a numerical simulation (solid line), and Eq. 47 with $\beta=2.5$ (dashed-dotted line)

quadratic curing function. The quadratic curing function is obtained by extending the linear curing function, Eq. 5, with a quadratic term:

$\hat{g}_{*}^{q}(t)=1+\xi_{E}^{l} t+\beta\left(\xi_{E}^{l}\right)^{2} t^{2}$

where the term $\beta\left(\xi_{E}^{l}\right)^{2}$ may be interpreted as the "curing acceleration", with $\beta$ being a dimensionless calibration parameter. In accordance with the coordinate transformation given by Eqs. 7 and 8, the quadratic curing function can be expressed in terms of the dimensionless Eulerian coordinate $\bar{X}$ as:

$\hat{\bar{g}}_{*}^{q}(\bar{X})=1-\bar{X}+\beta \bar{X}^{2} \quad$ with $\quad \bar{X}=\frac{\xi_{E}^{l} X}{i}$.

The numerical solution representing the buckling curve for the quadratic curing function (solid line) has been calculated by substituting Eq. 46 , using $\beta=2.5$, into the weak form of the homogeneous form of the equilibrium equation, Eq. 13, and the boundary conditions, Eqs. 21 and 22 , and solving these equations by applying the combined analytical-numerical solution procedure described in [21]. The numerical curve presented in Fig. 16 can be accurately approximated by extending the closed-form buckling curve for linear curing, Eq. 24, as:

$$
\begin{aligned}
& \bar{l}_{c r}=\bar{l}_{c r, 0}+c\left(\bar{\xi}_{E}^{l}\right)^{m}, \\
& \text { with } \quad c=\hat{c}(\beta)=0.996+2.328 \beta+0.268 \beta^{2}, \\
& \text { and } \quad m=\hat{m}(\beta)=0.793+1.300(1-\exp (-2.762 \beta)),
\end{aligned}
$$


where $\bar{l}_{c r, 0}=1.98635$. This closed-form expression has been found from calibrating the buckling curves computed numerically with the parametric model in the range of $0 \leq \beta \leq 5.0$, whereby the $R^{2}$ value varies between 0.982 and 0.997 for a curing rate ranging up to $\bar{\xi}_{E}^{l}=1.0$. It can be easily confirmed that for $\beta=0$ Eq. 47 indeed reduces to the buckling curve for linear curing, Eq. 24 . Figure 16 shows that for relatively low dimensionless curing rates, in the range of $0 \leq \bar{\xi}_{E}^{l} \leq 0.2$, the buckling curves related to the linear and quadratic curing functions are in close correspondence, and provide a good estimate for the experimental buckling length measured at $\bar{\xi}_{E}^{l}=0.062$. Specifically, the experimental buckling length corresponds to 21 layers, whereas the parametric model predicts buckling to occur at 19 layers, which results in a relative difference of $10 \%$. This accuracy is similar to that found in the experimental model validation presented in [21] for wall configurations printed at a comparable curing rate. For higher dimensionless curing rates, $\bar{\xi}_{E}^{l}>0.2$, Fig. 16 shows that the buckling curves for the linear and quadratic curing functions tend to diverge, whereby the buckling curve for linear curing strongly underestimates the experimental buckling lengths measured at $\bar{\xi}_{E}^{l}=0.312$ and 0.650 . Conversely, the buckling curve for quadratic curing, which accounts for an increase in curing rate with time, closely matches these two experimental values, whereby the relative difference with each experimental value is about $7 \%$. The reason for the increase in curing rate can be sought in the temperature rise induced during the relatively long printing session; specifically, due to the process of continuously mixing and pumping the cementitious material, the 3D concrete printing system heats up substantially over time. Heat generation speeds up the chemical reaction, which enlarges the curing rate and thereby the elastic stiffness of the material, thus leading to a larger buckling length compared to the case whereby the curing rate remains constant.

The effect of heat generation on the initial material stiffness can be demonstrated by comparing the results of ultrasound pulse velocity measurements on samples of fresh concrete (i.e. the material state as it comes out of the printing nozzle) taken at the beginning and the end of the printing sessions. The result of these measurements is depicted in Fig. 17, illustrating that after about $20 \mathrm{~min}$ of printing the pulse velocities-and thus the elastic stiffnesses-of the two fresh concrete samples start to diverge. Comparing this period to the total printing times of the walls with $b=5.0$ $\mathrm{m}(21.6 \mathrm{~min})$ and $b=10.4 \mathrm{~m}(76.5 \mathrm{~min})$ makes it indeed plausible that the buckling lengths measured for these walls are influenced by an increasing curing rate caused by thermal heating of the 3D printing facility. Note, however, that the thermal boundary conditions of the sample in the ultrasound pulse velocity tests differ from those of a wall in

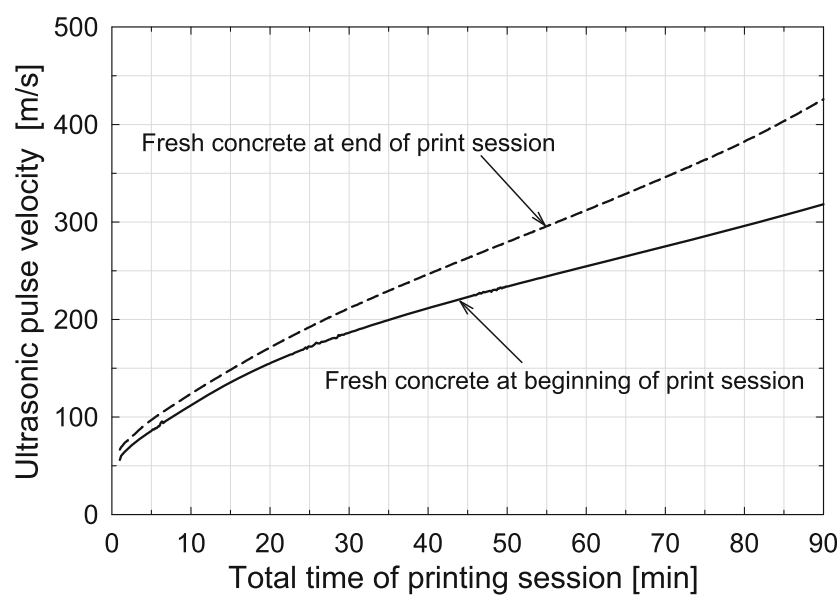

Fig. 17 Ultrasonic pulse velocity versus total time of printing session for samples representative of the fresh concrete deposited at the beginning (solid line) and end (dashed line) of a printing session

the $3 \mathrm{D}$ printing facility, from which it is concluded that the outcome of the ultrasone tests only provides a qualitative indication of the heat effect on the curing rate; a quantitative study of this effect is a topic for future research.

With the calibrated values of the initial stiffness, $E_{0}=$ $39.5 \mathrm{kPa}$, and the parameters for the quadratic curing function, $\xi_{E}^{l}=7.2 \times 10^{-4} \mathrm{~s}^{-1}$ and $\beta=2.5$, a combination of Eqs. 4 and 45 illustrates that for the case $b=10.4$ $\mathrm{m}$ the stiffness modulus at the bottom of the wall obtains a maximum value of $E_{*}=1.2 \mathrm{MPa}$ at the end of the printing process (i.e. after $76.5 \mathrm{~min}$ ). Note that this value is still very small compared to the stiffness modulus of a fully cured concrete, which is in the order of $10^{4}-10^{5} \mathrm{MPa}$. Nevertheless, it is about a factor of 7 higher than the value of $E_{*}=0.17 \mathrm{MPa}$ depicted in Fig. 14 for a linear curing process at $76.5 \mathrm{~min}$.

\section{Conclusions}

This contribution studies failure by elastic buckling and plastic collapse of wall structures during the process of extrusion-based 3D printing. Results obtained by the parametric 3D printing model recently developed by Suiker [21], which include closed-form expressions useful for engineering practice, are validated against results of dedicated FEM simulations and 3D concrete printing experiments. In the comparison with FEM simulations, the configurations analysed are a free wall (i.e. a wall without support along its vertical edges), a simply supported wall, a fully clamped wall and a rectangular wall layout. Two types of time-dependent curing processes of the printing material are considered, namely linear curing and exponentially decaying curing, which are subjected to three different curing rates (or printing velocities). The predictions of 
the critical buckling lengths of the free wall and the simply supported wall are in excellent agreement with the values following from the FEM simulations. For the fully clamped wall, the relative difference in the predicted critical buckling length is small at a low curing rate, but somewhat grows when the curing rate increases. This difference can be ascribed to the cosine approximation adopted in the parametric model for the horizontal buckling shape of the fully clamped wall, which starts to lose its accuracy at growing distance from the bottom of the wall. For a rectangular wall layout, the critical buckling lengths computed by the parametric model and FEM simulations are in close correspondence. The effect of imperfections on the buckling behaviour of the free wall is also similar for the two models, and indicates that the response computed for different imperfection wavelengths under increasing horizontal wall top deflection correctly approaches the corresponding bifurcation buckling length. Further, under a specific change of the material properties, the parametric and FEM models predict a similar transition in failure mechanism, from elastic buckling to plastic collapse.

For the validation of the parametric model with 3D concrete printing experiments, free walls were manufactured at three different widths, which allows for studying the effect of the curing rate (or printing velocity) on the wall buckling response. The buckling length measured at the smallest curing rate agrees well with the prediction by the parametric model under the application of a linear curing function. For the two experiments performed at higher curing rates, the model predictions based on a linear curing function lose their accuracy, which can be solved by extending the linear curing function with a quadratic term. The use of a quadratic curing function can be motivated from the observation that accelerated curing takes place as a result of thermal heating of the 3D printing facility under relatively large printing times. This thermal effect is relevant for most extrusionbased 3D printing processes, and therefore is an interesting topic for future research.

In correspondence with the above results, the present validation study confirms that the parametric $3 \mathrm{D}$ printing model presented in [21] provides a useful research and design tool for the prediction of failure of straight wall structures during the process of extrusion-based 3D printing. The model can be applied to quickly and systematically explore the influence of individual printing process parameters on the failure response of 3D-printed walls, which can be translated to directives regarding the optimisation of material usage and printing time. For the analysis of structures with more complicated geometries than the straight wall structures examined in this communication, the equations following from a parametric modelling approach may easily become relatively complex and unmanageable; hence, for such geometries, the accurate modelling of $3 \mathrm{D}$ printing processes requires the use of FEM modelling, as, for example, is done for the cylindrical wall structures analysed in [24].

Open Access This article is distributed under the terms of the Creative Commons Attribution 4.0 International License (http:// creativecommons.org/licenses/by/4.0/), which permits unrestricted use, distribution, and reproduction in any medium, provided you give appropriate credit to the original author(s) and the source, provide a link to the Creative Commons license, and indicate if changes were made.

\section{References}

1. Dimitrov D, Schreve K, de Beer N (2006) Advances in threedimensional printing - state of the art and future perspectives. Rapid Prototyp J 12(3):136-147

2. Williams CB, Mistfree F, Rosen DW (2011) A functional classification framework for the conceptual design of additive manufacturing technologies. J Mech Des 133(12):121002

3. Gross BC, Erkal JL, Lockwood SY, Chen C, Spence DM (2014) Evaluation of 3D printing and its potential impact on biotechnology and the chemical sciences. Anal Chem 86(7):32403253

4. Masood SH (2014) Advances in fused deposition modelling. In: Comprehensive Materials Processing. Elsevier, pp 69-91

5. Gao W, Zhang Y, Ramanujan D, Ramani K, Chenc Y, Williams CB, Wang CCL, Shin YC, Zhang S, Zavattieri PD (2015) The status, challenges, and future of additive manufacturing in engineering. Comput Aided Des 69:65-89

6. Chia HN, Wu BM (2015) Recent advances in 3D printing of biomaterials. J Biol Eng 9:4

7. Stansbury JW, Idacavage MJ (2016) 3D printing with polymers: Challenges among expanding options and opportunities. Dent Mater 32:54-64

8. Greul M, Pintat T, Greulich M (1995) Multiphase jet solidification (MJS). A rapid prototyping process for functional metallic and ceramic parts. European Conference on Advanced PM materials, Birmingham, pp 590-596

9. Vaidyanathan R, Walish J, Lombardi JL (2000) The extrusion freeforming of functional ceramic properties. J Miner Metals Mater Soc (TMS) 52(12):34

10. Grida I, Evans JRG (2003) Extrusion freeforming of ceramics through fine nozzles. J Eur Ceram Soc 23:629-635

11. Brooks H, Lupeanu ME, Piorkowski B (2013) Research towards high speed extrusion freeforming. Int J Rapid Manuf 3(2/3):154171

12. Khoshnevis SL, Bukkapatnam S, Kwon S, Saito J (2001) Experimental investigation of contour crafting using ceramic materials. Rapid Prototyp J 7(1):32-41

13. Feilden E, Garcia-Tuñón Blanca E, Giuliani F, Saiz E, Vandeperre L (2016) Robocasting of structural ceramic parts with hydrogel links. J Eur Ceram Soc 36(10):2525-2533

14. Liu CB, Gao JM, Tang YB, Chen XM (2018) Preparation and characterization of gypsum-based materials used for robocasting. J Mater Sci 53(24):16415-16422

15. Shahab AR, Kristensen E, Fisher P, Gramazio F, Kohler M, Flatt RJ (2013) Smart dynamic casting or how to exploit the liquid to solid transition in cementitious material. International conference of self-compacting concrete and 1st RILEM international conference on rheology and processing of construction materials, Paris France 
16. Schulteiss M, Wangler T, Reiter L, Roussel N, Flatt R (2016) Feedback control of smart dynamic casting through formwork friction measurements. In: 8th international RILEM symposium on self-compacting concrete, Washington DC

17. Boparai KS, Singh R, Chohan JS (2019) Fused deposition modelling: applications and advancements. In: Singh R, Paulo Davim J (eds) Additive Manufacturing: Applications and Innovations. CRC Press, Taylor \& Francis Group

18. Schubert C, van Langeveld MC, Donoso LA (2014) Innovations in 3D printing: a 3D overview from optics to organs. Br J Ophthalmol 98(2):159-161

19. Godoi FC, Prakash S, Bhandari BR (2016) 3D printing technologies applied for food design: Status and prospects. J Food Eng 179:44-54

20. Bos F, Wolfs R, Ahmed Z, Salet T (2016) Additive manufacturing of concrete in construction: potentials and challenges of $3 \mathrm{D}$ concrete. Virtual Phys Prototyp 11:209-225

21. Suiker ASJ (2018) Mechanical performance of wall structures in 3D printing processes: Theory, design tools and experiments. Int J Mech Sci 137:145-170

22. Perrot A, Rangeard D, Pierre A (2016) Structural built-up of cement-based materials used for 3D-printing extrusion techniques. Mater Struct 49(4):1213-1220

23. Wangler T, Lloret E, Reiter L, Hack N, Gramazio F, Kohler M, Bernhard M, Dillenburger B, Buchli J, Roussel N, Flatt R (2016) Digital concrete: oppurtunities and challenges. RILEM Techn Lett 1:67-75

24. Wolfs RJM, Bos FP, Salet TAM (2018) Early age mechanical behaviour of 3D printed concrete: Numerical modeling and experimental testing. Cem Concr Res 106:103-116

25. Roussel N (2018) Rheological requirements for printable concrete. Cem Concr Res 112:76-85

26. Benedetti A, Fernandes P, Granja JL, Sena-Cruz J, Azenha M (2016) Infleunce of temperature on the curing of an epoxy adhesive and its influence on bond behaviour of NSM-CFRP systems. Compos Part B - Eng 89:219-229

27. Ware HOT, Farsheed AC, van Lith R, Baker E, Ameer G, Sun C (2017) Process development for high-resolution 3Dprinting of bioresorbable vascular stents. In: von Freymann G, Schoenfeld WV, Rumpf RC (eds) Advanced Fabrication Technologies for Micro/Nano Optics and Photonics, Proceedings of SPIE, vol 10115, pp 10115N1-11

28. Bhutta MAR (2010) Effect of polymer-cement ratio and accelerated curing on flexural behavior of hardener-free epoxymodified mortar panels. Mater Struct 43:429-439

29. Zhou Y-G, Su B, Turng L-S (2017) Deposition-induced effects of isotactic polypropylene and polycarbonate composites during fused deposition modelling. Rapid Prototyp J 23:869-880

30. Thrimurthulu K, Pandey PM, Reddy NV (2004) Optimum part deposition orientation in fused deposition modelling. Int $\mathrm{J}$ Mach Tools Manuf 44:585-594

31. Turner BN, Strong R, Gold SA (2014) A review of melt extrusion additive manufacturing processes: I Process design and modelling. Rapid Prototyp J 20:192-204

32. Wijffels MJH, Wolfs RJM, Suiker ASJ, Salet TAM (2017) Magnetic orientation of steel fibres in self-compacting concrete beams: Effect on failure behaviour. Cem Concr Compos 80:342355

33. Wolfs RJM, Bos FP, Salet TAM (2019) Triaxial compression testing on early age concrete for numerical analysis of 3D concrete printing. Cem Concr Compos. https://doi.org/10. 1016/j.cemconcomp.2019.103344

34. Standard Test Method for Unconfined Compressive Strength of Cohesive Soil (2013) ASTM D2166

Publisher's note Springer Nature remains neutral with regard to jurisdictional claims in published maps and institutional affiliations. 